\title{
Diastereoselective Aldol Additions of Chiral $\beta$-Hydroxy Ethyl Ketone Enolates Catalyzed by Lewis Bases
}

\author{
Scott E. Denmark* and Shinji Fujimori \\ Roger Adams Laboratory, Department of Chemistry, University of Illinois, \\ Urbana, Illinois 61801
}

\section{SUPPORTING INFORMATION}

\section{General Experimental}

${ }^{1} \mathrm{H},{ }^{13} \mathrm{C}$ and ${ }^{31} \mathrm{P}$ NMR spectra were recorded on Varian Unity-400 $\left(400 \mathrm{MHz}{ }^{1} \mathrm{H}, 100\right.$ $\left.\mathrm{MHz}{ }^{13} \mathrm{C}, 162 \mathrm{MHz}{ }^{31} \mathrm{P}\right)$, Unity-500 (500 MHz $\left.{ }^{1} \mathrm{H}, 125 \mathrm{MHz}{ }^{13} \mathrm{C}, 202 \mathrm{MHz}{ }^{31} \mathrm{P}\right)$ and Varian Unity Inova $500\left(500 \mathrm{MHz}{ }^{1} \mathrm{H}\right)$ spectrometers in deuterochloroform unless otherwise stated. Data are reported in the following order: chemical shift in ppm $(\delta)$; multiplicities are indicated (br (broadened), s (singlet), d (doublet), t (triplet), q (quartet), m (multiplet)); coupling constants, $J$, are reported in hertz $(\mathrm{Hz})$; integration is provided; and assignment is indicated. Mass spectrometry was performed by the University of Illinois Mass Spectrometry Center. Electron impact (EI) spectra were performed on a Finnigan-MAT CH-5 spectrometer, chemical ionization (CI) spectra were obtained on a VG 70-VSE spectrometer using methane as the carrier gas, and field ionization (FI) spectra were obtained from VG 70-VSE A spectrometer. Data are reported in the form of $\mathrm{m} / \mathrm{z}$ (intensity relative to base peak =100). Infrared spectra (IR) were recorded on a Mattson Galaxy 5020 spectrophorometer. Peaks are reported in $\mathrm{cm}^{-1}$ with indicated intensities: s (strong, $67-100 \%$ absorption), m (medium, $34-66 \%$ ), w (weak, $0-33 \%$ ). Optical rotations were measured on a Jasco DIP-360 digital polarimeter and are reported in the standard format for $[\alpha]$. Elemental analyses were performed by the University of Illinois Microanalytical Service Laboratory.

Analytical thin-layer chromatography was performed on Merck silica gel plates with F254 indicator. Visualization was accomplished by UV light, iodine, potassium permanganate solution $\left(\mathrm{KMnO}_{4}\right)$, phosphomolibdic acid stain(PMA), p-anisaldehyde stain or dinitrophenylhydrazine (DNP) solution. Column chromatography was performed by the method 
of Still ${ }^{1}$ with 32-63 mm silica gel (Merck). Bulb-to-bulb distillations were performed on a Buchi GKR-50 Kugelrohr and boiling points (bp) correspond to uncorrected, air bath temperatures. Analytical supercritical fluid chromatography (SFC) was performed on a Berger Instruments packed-column SFC with built in photometric detector. Analytical columns used were Daicel Chiralpak $\mathrm{AD}$ and $\mathrm{AS}$, Chiralcel OJ and OD and Regis $(R, R)$-Welk-01. Melting points (mp) were determined on a Thomas-Hoover melting point apparatus and are uncorrected.

All reactions were performed in oven $\left(140{ }^{\circ} \mathrm{C}\right)$ and/or flame-dried glassware under an atmosphere of dry nitrogen, unless otherwise stated. Solvents for extraction and chromatography were technical grade and distilled from the indicated drying agents: dichloromethane $\left(\mathrm{CH}_{2} \mathrm{Cl}_{2}\right)$, pentane, hexane: $\mathrm{CaCl}_{2}$; ethyl acetate (EtOAc): $\mathrm{K}_{2} \mathrm{CO}_{3}$; acetone and methanol $(\mathrm{MeOH})$ were reagent grade. $n$-Butyllithium was titrated according to the method of Gilman. 2 "Brine" refers to a saturated solution of $\mathrm{NaCl}$.

\section{Literature Preparations}

The following compounds were prepared by literature methods: $(S)-N$-Methoxy- $N$ methyl-3-hydroxy-2-methylpropionamide $\quad(9)^{3}$, (S)-N-Methoxy- $N$-methyl-3-triisopropylsilanyloxy-2-methylpropionamide (12) ${ }^{4}, \quad(S)-N$-Methoxy- $N$-methyl-3-(tert-butyldimethylsilanyloxy)-2-methylpropionamide $\quad(\mathbf{1 1})^{5}, \quad(S)$-1-triisopropylsilanyloxy-2-methyl-3pentanone $(\mathbf{4})^{4}$

The enolisation using LiTMP followed the procedure described by Collum et al. ${ }^{6}$ The enolization using DBU to synthesize $(Z)$-enol ethers followed the procedure described by Yamaguchi, et. al. ${ }^{7}$ tert-Butyltritylamine (TBTA) was prepared according to the procedure described by Corey, et. al. ${ }^{8}$ 


\section{Experimental Procedures}

\section{(S)-1-tert-Butyldimethylsilyloxy-2-methyl-3-pentanone (3)}

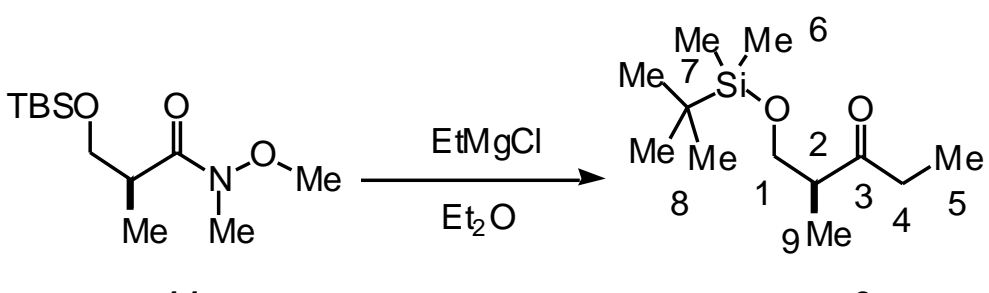

11

\section{3}

Amide 11 (9.50 g, $36.4 \mathrm{mmol}$ ) was dissolved in $100 \mathrm{~mL}^{\circ} \mathrm{Et}_{2} \mathrm{O}$ at $0{ }^{\circ} \mathrm{C}$. To this solution was added slowly a solution of ethyl magnesium chloride $\left(2.0 \mathrm{M}\right.$ in $\mathrm{Et}_{2} \mathrm{O}, 21.0 \mathrm{~mL}, 41.9 \mathrm{mmol}$, 1.15 equiv). The reaction mixture was stirred at $0{ }^{\circ} \mathrm{C}$ for $1 \mathrm{~h}$, then was gradually warmed up to $\mathrm{rt}$ over $11 \mathrm{~h}$. The reaction mixture was quenched by slow addition of sat. aq. ammonium chloride solution (ca. $50 \mathrm{~mL}$ ). The layers were separated and the aqueous layer was extracted twice with $100 \mathrm{~mL}$ of $\mathrm{Et}_{2} \mathrm{O}$. The extracts were combined, dried over $\left(\mathrm{MgSO}_{4}\right)$, filtered, and concentrated to give $9.68 \mathrm{~g}$ of an oil. The crude product was purified by chromatography (pentane/ether, 3/1, $\left.\mathrm{SiO}_{2}\right)$ and distillation to afford $7.84 \mathrm{~g}$ of $3(34.0 \mathrm{mmol}, 93 \%)$ as a clear, colorless liquid.

Data for 3:

bp: $\quad 64^{\circ} \mathrm{C}(0.5 \mathrm{mmHg})$

1ㅅN: $\quad\left(500 \mathrm{MHz}, \mathrm{CDCl}_{3}\right)$

3.74-3.58 (ABX, $\left.2 \mathrm{H}, \mathrm{H}_{2} \mathrm{C}(1)\right)$ ) 2.78 (sext, $\left.J=6.1,1 \mathrm{H}, \mathrm{HC}(2)\right)$ ) $2.52(\mathrm{ABX}, 2 \mathrm{H}$, $\left.\mathrm{H}_{2} \mathrm{C}(4)\right) ; 1.03$ (t, $\left.J=7.1,3 \mathrm{H}, \mathrm{H}_{3} \mathrm{C}(5)\right) ; 1.02$ (d, $\left.J=7.1,3 \mathrm{H}, \mathrm{H}_{3} \mathrm{C}(9)\right) ; 0.86$ (s, 9 $\left.\mathrm{H}, \mathrm{H}_{3} \mathrm{C}(8)\right) ; 0.03\left(\mathrm{~d}, J=7.1,6 \mathrm{H}, \mathrm{H}_{3} \mathrm{C}(6)\right)$

${ }^{13}$ C NMR: $\quad\left(126 \mathrm{MHz}, \mathrm{CDCl}_{3}\right)$

214.70 (C(3)); 65.93 (C(1)); 48.49 (C(2)); 36.17 (C(4)); 25.99 (C(8)); 18.37 $(\mathrm{C}(7)) ; 13.28(\mathrm{C}(9)) ; 7.65(\mathrm{C}(5)) ;-5.38,-5.40(\mathrm{C}(6))$

IR: (neat)

2956 (s); 2932 (s); 2886 (m); 2859 (m); 1718 (s); 1463 (m); 1388 (w); $1361(\mathrm{w})$; 1257 (m); 1092 (s)

MS: (FI)

231.1 (1); $230.1\left(1, \mathrm{M}^{+}\right) ; 175.1(4) ; 174.1(14) ; 173.1(100) ; 122.1$ (1) 
Opt. Rot.: $\quad[\alpha]_{\mathrm{D}}^{24}+43.70\left(\mathrm{c}=1.0, \mathrm{CHCl}_{3}\right)$

TLC: $\quad R_{f} 0.28$ (hexane/EtOAc, 10/1) [silica gel, $\mathrm{KMnO}_{4}$ ]

$R_{f} 0.53$ (pentane/ether, 3/1) [silica gel, $\mathrm{KMnO}_{4}$ ]

Analysis: $\quad \mathrm{C}_{12} \mathrm{H}_{26} \mathrm{O}_{2} \mathrm{Si}(230.42)$
Calcd:
C, 62.55;
H, $11.37 \%$
Found:
C, 62.29;
H, $11.52 \%$

\section{$(2 Z, 4 S)-5$-(tert-Butyldimethylsilyloxy)-4-methyl-3-trimethylsilyloxy-2-pentene}

((Z)-13)

(Using triethylamine/TMSOTf)

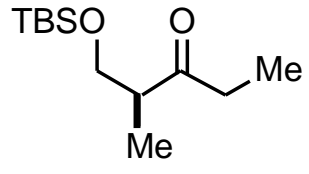

3

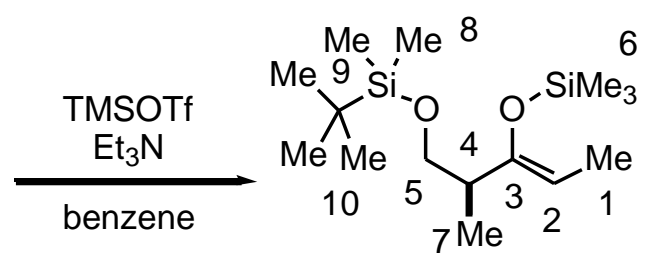

(Z)-13

Trimethylsilyl triflate (TMSOTf, $0.93 \mathrm{~mL}, 5.17 \mathrm{mmol}, 1.2$ equiv) was dissolved in $15 \mathrm{~mL}$ of benzene at $0{ }^{\circ} \mathrm{C}$. To the solution was added triethylamine $(0.88 \mathrm{~mL}, 6.45 \mathrm{mmol}, 1.5$ equiv) and 3 (990 $\mathrm{mg}, 4.30 \mathrm{mmol})$. The reaction mixture was allowed to warm to $\mathrm{rt}$ and was stirred for $5 \mathrm{~h}$. The reaction mixture was cooled using an ice bath and $15 \mathrm{~mL}$ of cold water was added. The product was extracted with $45 \mathrm{~mL}$ of pentane, and the pentane extracts were washed with sat. aq. $\mathrm{CuSO}_{4}$ solution, $10 \mathrm{~mL}$ of water, and $10 \mathrm{~mL}$ of brine. The organic layer was dried $\left(\mathrm{Na}_{2} \mathrm{SO}_{4}\right)$, filtered, and concentrated to yield $1.42 \mathrm{~g}$ of an oil. The crude product was purified by chromatography (hexane/ $\left.\mathrm{CH}_{2} \mathrm{Cl}_{2}, 8 / 1, \mathrm{SiO}_{2}\right)$ to afford $100 \mathrm{mg}$ of $(E)-\mathbf{1 3}(0.33 \mathrm{mmol}, 8 \%)$ and $688 \mathrm{mg}$ of (Z)-13 (2.27 mmol, 53\%) both as clear, colorless oils. Ether (Z)-13 was further purified by Kugelrohr distillation.

Data for $(Z)-\mathbf{1 3}$ :

bp: $\quad 170{ }^{\circ} \mathrm{C}(15 \mathrm{mmHg}, \mathrm{ABT})$ 
${ }^{1} \underline{\mathrm{H} \text { NMR: }} \quad\left(500 \mathrm{MHz}, \mathrm{CDCl}_{3}\right)$

4.53 ( qd, $J=6.9,0.8,1 \mathrm{H}, \mathrm{HC}(2)$ ); 3.75-3.30 (ABX, $2 \mathrm{H}, \mathrm{H}_{2} \mathrm{C}(5)$ ); 2.20 (sext, $J$ $=6.1,1 \mathrm{H}, \mathrm{HC}(4)) ; 1.50\left(\mathrm{dd}, J=6.8,0.9,3 \mathrm{H}, \mathrm{H}_{3} \mathrm{C}(3)\right) ; 1.02(\mathrm{~d}, J=6.8,3 \mathrm{H}$, $\mathrm{H}_{3} \mathrm{C}(6)$ ); 0.88 (s, $9 \mathrm{H}, \mathrm{H}_{3} \mathrm{C}(10)$ ); 0.20 (s, $9 \mathrm{H}, \mathrm{TMS}$ ); 0.03 (s, $6 \mathrm{H}, \mathrm{H}_{3} \mathrm{C}(8)$ )

${ }^{13} \mathrm{C} \mathrm{NMR}: \quad\left(126 \mathrm{MHz}, \mathrm{CDCl}_{3}\right)$

$152.85(\mathrm{C}(3)) ; 101.64(\mathrm{C}(2)) ; 66.40(\mathrm{C}(5)) ; 43.06(\mathrm{C}(4)) ; 25.90(\mathrm{C}(10)) ; 18.30$ $(\mathrm{C}(9)) ; 15.26(\mathrm{C}(7)) ; 10.91(\mathrm{C}(1)) ; 0.70(\mathrm{C}(6)) ;-5.40(\mathrm{C}(8))$

IR: (neat) 2958 (s); 2931 (s); 2860 (m); 1675 (m); 1472 (m); 1389 (w); $1319(\mathrm{w}) ; 1254$ (s); 1191 (m); 1087 (s)

MS: $\quad(\mathrm{FI})$

305.1 (2); 304.1 (12); 303.1 (31); 302.1 (100, M+); 245.0 (2); 198.8 (2

Opt. Rot.: $\quad[\alpha]_{\mathrm{D}}^{24}+1.50\left(\mathrm{c}=1.0, \mathrm{CHCl}_{3}\right)$

TLC: $\quad R_{f} 0.25$ (hexane/ $\left.\mathrm{CH}_{2} \mathrm{Cl}_{2}, 5 / 1\right)$ [silica gel, $\mathrm{KMnO}_{4}$ ]

Analysis: $\quad \mathrm{C}_{15} \mathrm{H}_{34} \mathrm{O}_{2} \mathrm{Si}_{2}(302.60)$

Calcd: $\quad$ C, 59.54; $\quad \mathrm{H}, 11.33 \%$

Found: $\quad$ C, 59.27 ;, $11.52 \%$

(2E,4S)-5-(tert-Butyldimethylsilyloxy)-4-methyl-3-trimethylsilyloxy-2-pentene

((E)-13) (Using TBTA/TMSCl)

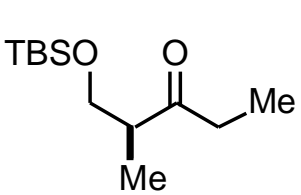

3

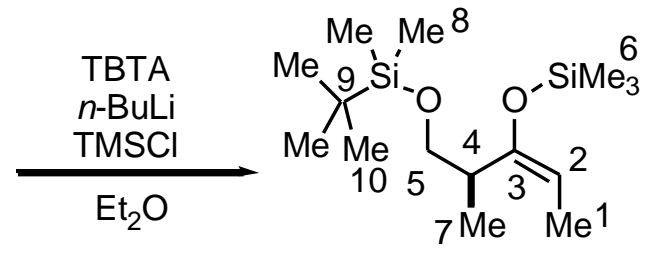

$(E)-13$

To a solution of tert-butyltritylamine $(2.05 \mathrm{~g}, 6.51 \mathrm{mmol}, 1.5$ equiv) in $30 \mathrm{~mL}$ of THF at $0{ }^{\circ} \mathrm{C}$ was added dropwise a solution of $n$-BuLi $(1.54 \mathrm{M}$ in hexane, $3.95 \mathrm{~mL}, 6.08 \mathrm{mmol}, 1.4$ equiv). The solution was stirred at $0{ }^{\circ} \mathrm{C}$ for $10 \mathrm{~min}$ then was cooled to $-78{ }^{\circ} \mathrm{C}$ and $\mathrm{TMSCl}(0.83$ $\mathrm{mL}, 6.51 \mathrm{mmol}, 1.5$ equiv) was added. A solution of ketone 3 in $5 \mathrm{~mL}$ of THF (1.00 g, 4.34 
mmol) was added to the reaction mixture via a cannula. The reaction mixture was stirred for 30 min, then was gradually warmed to $0{ }^{\circ} \mathrm{C}$ and was poured into $15 \mathrm{~mL}$ of cold, deionized water. The layers were separated and the aqueous layer was extracted with $50 \mathrm{~mL}$ of pentane. The combined organic layers were washed with $20 \mathrm{~mL}$ of sat. aq. $\mathrm{CuSO}_{4}$ solution, $10 \mathrm{~mL}$ of water, and $10 \mathrm{~mL}$ of brine, then were dried $\left(\mathrm{Na}_{2} \mathrm{SO}_{4}\right)$, filtered, and concentrated. The crude product was purified by Kugelrohr distillation $\left(175^{\circ} \mathrm{C} \mathrm{ABT}\right.$ at $\left.15 \mathrm{mmHg}\right)$ and column chromatography (hexane $\left./ \mathrm{CH}_{2} \mathrm{Cl}_{2}, 5 / 1, \mathrm{SiO}_{2}\right)$ to afford $727 \mathrm{mg}$ of $(E)-13(2.40 \mathrm{mmol}, 55 \%)$ as a clear, colorless oil and $68 \mathrm{mg}$ of $\mathbf{1 3}$ (0.22 mmol, 5\%, 1/1, Z/E mixture) as colorless oil.

Data for $(E)-13:$

bp: $\quad 150{ }^{\circ} \mathrm{C}(1.0 \mathrm{mmHg}, \mathrm{ABT})$

${ }^{1} \underline{\mathrm{H} \mathrm{NMR}}: \quad\left(500 \mathrm{MHz}, \mathrm{CDCl}_{3}\right)$

4.58 ( q, $J=6.8,1 \mathrm{H}, \mathrm{HC}(2)$ ); 3.60-3.38 (ABX, $2 \mathrm{H}, \mathrm{H}_{2} \mathrm{C}(5)$ ); 2.73 (sext, $J=7.1$, $1 \mathrm{H}, \mathrm{HC}(4)) ; 1.55$ (d, $\left.J=6.8,3 \mathrm{H}, \mathrm{H}_{3} \mathrm{C}(3)\right) ; 0.93$ (d, $\left.J=6.9,3 \mathrm{H}, \mathrm{H}_{3} \mathrm{C}(6)\right) ; 0.88$ (s, 9 H); 0.20 (s, 9 H, TMS); 0.03 (s, $6 \mathrm{H})$

${ }^{13}$ C NMR: $\quad\left(126 \mathrm{MHz}, \mathrm{CDCl}_{3}\right)$

$153.03(\mathrm{C}(3)) ; 100.27(\mathrm{C}(2)) ; 65.76(\mathrm{C}(5)) ; 36.69(\mathrm{C}(4)) ; 25.90(\mathrm{C}(10)) ; 18.29$ $(\mathrm{C}(9)) ; 14.07(\mathrm{C}(7)) ; 11.45(\mathrm{C}(1)) ; 0.39(\mathrm{C}(6)) ;-5.38,-5.41(\mathrm{C}(8))$

IR: (neat) 2958 (m); 2932 (m); 2898 (m); 2860 (m); 1667 (m); $1472(\mathrm{~m}) ; 1388(\mathrm{w}) ; 1363$ (w); 1253 (s); 1223 (s); 1105 (s); 1048 (m)

MS: $\quad(\mathrm{FI})$

304.1 (10); 303.1 (30); $302.1\left(100, \mathrm{M}^{+}\right) ; 199.0$ (2); 198.6 (2)

Opt. Rot.: $\quad[\alpha]_{\mathrm{D}}^{24}-8.99\left(\mathrm{c}=0.67, \mathrm{CHCl}_{3}\right)$

TLC: $\quad R_{f} 0.38$ (hexane/ $\left.\mathrm{CH}_{2} \mathrm{Cl}_{2}, 5 / 1\right)$ [silica gel, $\mathrm{KMnO}_{4}$ ]

Analysis: $\quad \mathrm{C}_{15} \mathrm{H}_{34} \mathrm{O}_{2} \mathrm{Si}_{2}(302.60)$
Calcd:
C, 59.54;
$\mathrm{H}, 11.33 \%$
Found:
C, 59.54;
$\mathrm{H}, 11.63 \%$ 


\section{$(2 Z, 4 S)$ - 5-(Triisopropylsilyloxy)-4-methyl-3-trimethylsilyloxy-2-pentene ((Z)-14)}

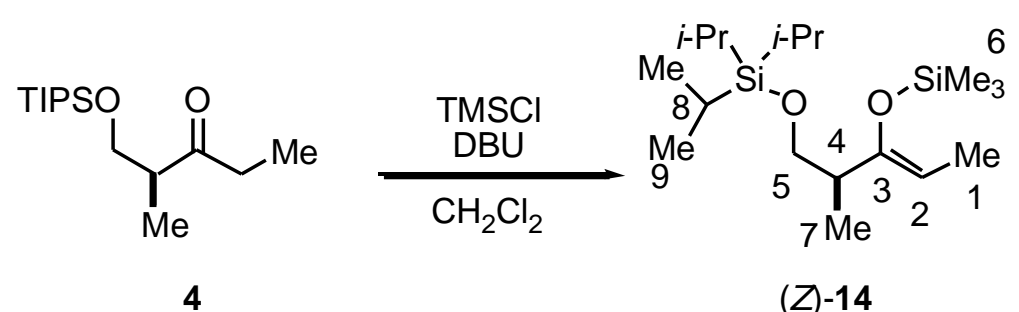

Trimethylsilyl chloride (TMSCl, $3.25 \mathrm{~mL}, 25.6 \mathrm{mmol}, 1.2$ equiv) was dissolved in $40 \mathrm{~mL}$ of $\mathrm{CH}_{2} \mathrm{Cl}_{2}$ at rt. To the solution was added DBU (3.84 mL, $25.6 \mathrm{mmol}, 1.2$ equiv) and 4 (5.80 g, $21.3 \mathrm{mmol}$ ). The reaction mixture was heated to reflux for $4 \mathrm{~h}$. The reaction mixture was cooled using an ice bath and $40 \mathrm{~mL}$ of cold, sat. aq. $\mathrm{NaHCO}_{3}$ solution was added. The product was extracted with $100 \mathrm{~mL}$ of pentane, and the pentane extracts were washed with $40 \mathrm{~mL}$ of brine. The organic layer was dried $\left(\mathrm{Na}_{2} \mathrm{SO}_{4}\right)$, filtered, and concentrated to afford $5.50 \mathrm{~g}$ of an oil. The crude product was purified by chromatography (pentane/ $\mathrm{CH}_{2} \mathrm{Cl}_{2}, 5 / 1, \mathrm{SiO}_{2}$ ) to afford $5.03 \mathrm{~g}$ of (Z)-14 (14.6 mmol, 69\%) as clear, colorless oil. Silyl ether $(Z)-\mathbf{1 4}$ was subsequently purified by Kugelrohr distillation.

Data for $(Z)-\mathbf{1 4}$ :

bp: $\quad 125^{\circ} \mathrm{C}(0.05 \mathrm{mmHg}, \mathrm{ABT})$

1슬 $\quad\left(500 \mathrm{MHz}, \mathrm{CDCl}_{3}\right)$

4.55 ( q, $J=6.7,1 \mathrm{H}, \mathrm{HC}(2))$; 3.85-3.38 (ABX, $\left.2 \mathrm{H}, \mathrm{H}_{2} \mathrm{C}(5)\right)$ ) 2.23 (sext, $J=6.3$,

$1 \mathrm{H}, \mathrm{HC}(4)) ; 1.51\left(\mathrm{~d}, J=6.6,3 \mathrm{H}, \mathrm{H}_{3} \mathrm{C}(3)\right) ; 1.10-1.03$ (m, $24 \mathrm{H}, \mathrm{H}_{3} \mathrm{C}(7)$, $\left.\mathrm{H}_{3} \mathrm{C}(8), \mathrm{HC}(8)\right) ; 0.19$ (s, 9 H, TMS);

${ }^{13}$ C NMR: $\quad\left(126 \mathrm{MHz}, \mathrm{CDCl}_{3}\right)$

153.19 (C(3)); $101.86(\mathrm{C}(2)) ; 66.97$ (C(5)); 43.58 (C(4)); 18.22 (C(9)); 15.55 $(\mathrm{C}(7)) ; 12.18(\mathrm{C}(8)) ; 11.14(\mathrm{C}(1)) ; 0.92(\mathrm{C}(6))$;

IR: (neat)

2958 (s); 2931 (s); 2860 (m); 1675 (m); 1472 (m); 1389 (w); 1319 (w); 1254 (s); $1191(\mathrm{~m}) ; 1087$ (s)

MS: (FI) 347.4 (2); 346.4 (12); $345.4(31) ; 344.4\left(100, \mathrm{M}^{+}\right)$; 
Opt. Rot.: $\quad[\alpha]_{\mathrm{D}}^{24}+4.18\left(\mathrm{c}=2.5, \mathrm{CHCl}_{3}\right)$

TLC: $\quad R_{f} 0.33$ (pentane/ $\mathrm{CH}_{2} \mathrm{Cl}_{2}, 5 / 1$ ) [silica gel, $\mathrm{KMnO}_{4}$ ]

Analysis: $\quad$ Calc for $\mathrm{C}_{18} \mathrm{H}_{40} \mathrm{O}_{2} \mathrm{Si}_{2}$ (344.68)
Calcd:
C, 62.72;
$\mathrm{H}, 11.70 \%$

Found:

C, 62.63;

H, $11.61 \%$

\section{General Procedure I: MeLi-mediated transilylation of 13: (2Z,4S)- 5-(tert-Butyl- dimethylsilyloxy)-4-methyl-3-trichlorosilyloxy-2-pentene $((Z)-5)$}

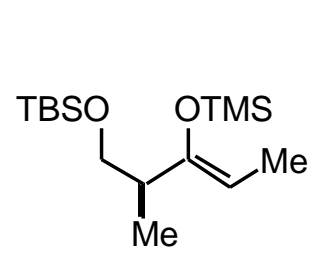

(Z)-13

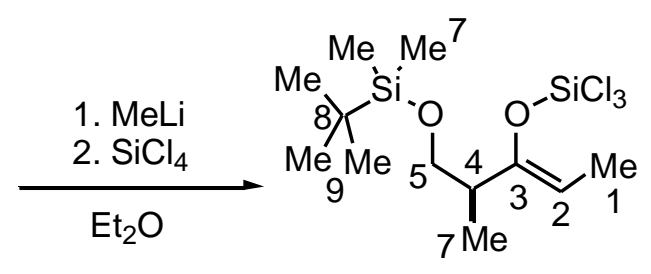

(Z)-5

Silyl enol ether $(Z)-\mathbf{1 3}(908 \mathrm{mg}, 3.00 \mathrm{mmol})$ was dissolved in $6 \mathrm{~mL}$ of $\mathrm{Et}_{2} \mathrm{O}$ at $0{ }^{\circ} \mathrm{C}$. To the solution was slowly added a solution of $\mathrm{MeLi}\left(1.5 \mathrm{M}\right.$ in $\mathrm{Et}_{2} \mathrm{O}, 3.00 \mathrm{~mL}, 4.50 \mathrm{mmol}, 1.5$ equiv). The reaction mixture was stirred for $4.5 \mathrm{~h}$ at $\mathrm{rt}$, then was cooled to $-78{ }^{\circ} \mathrm{C}$. The reaction mixture was transferred via cannula to a cold solution of silicon tetrachloride $(3.45 \mathrm{~mL}, 30.0$ mmol, 10 equiv) in $6 \mathrm{~mL}$ of $\mathrm{Et}_{2} \mathrm{O}$. The reaction mixture was stirred at $-78{ }^{\circ} \mathrm{C}$ for $1 \mathrm{~h}$ and was gradually allowed to warm to rt. The precipitate was allowed to settle to the bottom of the flask, and the supernatant was transferred to another flask and was concentrated in vacuo. The product was purified by Kugelrohr distillation to afford $901 \mathrm{mg}(2.48 \mathrm{mmol}, 83 \%)$ of (Z)-5 as a clear, colorless oil.

Data for $(Z)-\mathbf{5}$ :

bp: $\quad 150{ }^{\circ} \mathrm{C}(1.0 \mathrm{mmHg}, \mathrm{ABT})$

${ }^{1} \underline{\mathrm{H} \mathrm{NMR}}: \quad\left(\mathrm{CDCl}_{3}, 500 \mathrm{MHz}\right)$

$4.53(\mathrm{qd}, J=6.8,0.5,1 \mathrm{H}, \mathrm{HC}(2))$ ) 3.69-3.43 (ABX, $\left.2 \mathrm{H}, \mathrm{H}_{2} \mathrm{C}(5)\right)$ ) 2.40 (sext, $J=$ 6.3, $1 \mathrm{H}, \mathrm{HC}(4)) ; 1.60\left(\mathrm{dd}, J=7.1,0.7,3 \mathrm{H}, \mathrm{H}_{3} \mathrm{C}(1)\right) ; 1.07$ (d, $J=7.1,3 \mathrm{H}$, $\left.\mathrm{H}_{3} \mathrm{C}(6)\right) ; 0.88$ (s, $\left.9 \mathrm{H}, \mathrm{H}_{3} \mathrm{C}(9)\right) ; 0.03$ (s, $6 \mathrm{H}, \mathrm{H}_{3} \mathrm{C}(7)$ ) 
${ }^{13} \underline{\mathrm{C} \mathrm{NMR}}: \quad\left(\mathrm{CDCl}_{3}, 126 \mathrm{MHz}\right)$

$150.80(\mathrm{C}(3)) ; 106.42(\mathrm{C}(2)) ; 65.31(\mathrm{C}(5)) ; 42.11(\mathrm{C}(4)) ; 25.83(\mathrm{C}(9)) ; 18.22$ $(\mathrm{C}(8)) ; 14.85(\mathrm{C}(7)) ; 11.04(\mathrm{C}(1)) ;-5.46,-5.44(\mathrm{C}(7))$

(2E,4S)- 5-(tert-Butyldimethylsilyloxy)-4-methyl-3-trichlorosilyloxy-2-pentene $((E)-5)$

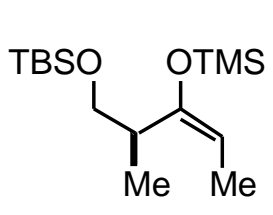

(E)-13

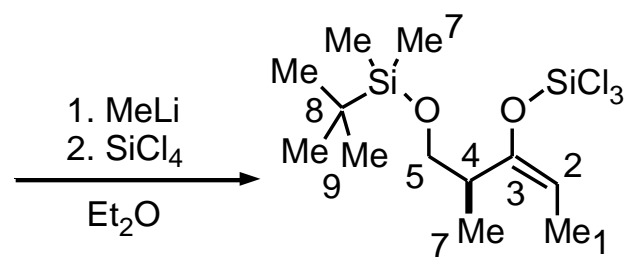

$(E)-5$

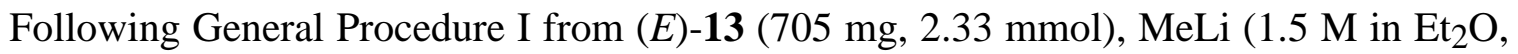
$2.33 \mathrm{~mL}, 3.49 \mathrm{mmol}, 1.5$ equiv) and $\mathrm{SiCl}_{4}$ (2.67 mL, $23.3 \mathrm{mmol}, 10$ equiv) in $7 \mathrm{~mL}$ of ether, was obtained $705 \mathrm{mg}(1.94 \mathrm{mmol}, 83 \%)$ of $(E)-5$ as a clear, colorless oil.

Data for $(E)-5$ :

bp: $\quad 150{ }^{\circ} \mathrm{C}(1.0 \mathrm{mmHg}, \mathrm{ABT})$

1슬 $: \quad\left(\mathrm{CDCl}_{3}, 500 \mathrm{MHz}\right)$

5.10 (q, $J=7.1,1 \mathrm{H}, \mathrm{HC}(2)) ; 3.60-3.43\left(\mathrm{ABX}, 2 \mathrm{H}, \mathrm{H}_{2} \mathrm{C}(5)\right)$ ) 2.83 (sext, $J=6.8$, $1 \mathrm{H}, \mathrm{HC}(4)) ; 1.61\left(\mathrm{~d}, J=6.8,3 \mathrm{H}, \mathrm{H}_{3} \mathrm{C}(1)\right) ; 0.99$ (d, $\left.J=6.8,3 \mathrm{H}, \mathrm{H}_{3} \mathrm{C}(6)\right) ; 0.88$ $\left(\mathrm{s}, 9 \mathrm{H}, \mathrm{H}_{3} \mathrm{C}(9)\right) ; 0.03\left(\mathrm{~d}, J=2.6,6 \mathrm{H}, \mathrm{H}_{3} \mathrm{C}(7)\right)$

${ }^{13}$ C NMR: $\quad\left(\mathrm{CDCl}_{3}, 126 \mathrm{MHz}\right)$

150.78 (C(3)); $106.67(\mathrm{C}(2)) ; 65.00(\mathrm{C}(5)) ; 36.40(\mathrm{C}(4)) ; 25.84(\mathrm{C}(9)) ; 18.22$ $(\mathrm{C}(8)) ; 13.63(\mathrm{C}(1)) ; 11.43(\mathrm{C}(6)) ;-5.47,-5.49(\mathrm{C}(7))$ 
(2Z,4S)- 5-(Triisopropylsilyloxy)-4-methyl-3-trichlorosilyloxy-2-pentene ((Z)-6)

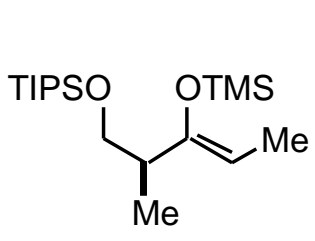

(Z)-14

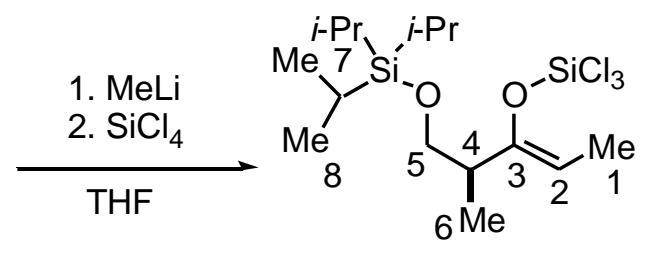

(Z)-6

Following General Procedure I from $(Z)-14(6.51 \mathrm{~g}, 18.9 \mathrm{mmol}), \mathrm{MeLi}\left(1.5 \mathrm{M}\right.$ in $\mathrm{Et}_{2} \mathrm{O}$, $18.9 \mathrm{~mL}, 28.4 \mathrm{mmol}, 1.5$ equiv) and $\mathrm{SiCl}_{4}(21.7 \mathrm{~mL}, 189 \mathrm{mmol}, 10$ equiv) in $50 \mathrm{~mL}$ of THF, was obtained $6.14 \mathrm{~g}(12.7 \mathrm{mmol}, 67 \%)$ of $(Z)-6$ as a clear, colorless oil.

Data for $(Z)-6$ :

bp: $\quad 115^{\circ} \mathrm{C}(1.0 \mathrm{mmHg}, \mathrm{ABT})$

${ }^{1} \underline{\mathrm{H} \mathrm{NMR}}: \quad\left(500 \mathrm{MHz}, \mathrm{CDCl}_{3}\right)$

$4.85(\mathrm{qd}, J=6.9,0.5,1 \mathrm{H}, \mathrm{HC}(2))$; 3.80-3.51 (ABX, $\left.2 \mathrm{H}, \mathrm{H}_{2} \mathrm{C}(5)\right) ; 2.43$ (sext, $J=$ 5.8, $1 \mathrm{H}, \mathrm{HC}(4)) ; 1.60\left(\mathrm{dd}, J=6.9,0.9,3 \mathrm{H}, \mathrm{H}_{3} \mathrm{C}(3)\right) ; 1.10(\mathrm{~d}, J=6.9,3 \mathrm{H}$, $\left.\mathrm{H}_{3} \mathrm{C}(6)\right) ; 1.10-1.00\left(\mathrm{~m}, 21 \mathrm{H}, \mathrm{H}_{3} \mathrm{C}(8), \mathrm{HC}(7)\right)$

${ }^{13} \underline{\mathrm{C} \mathrm{NMR}}: \quad\left(126 \mathrm{MHz}, \mathrm{CDCl}_{3}\right)$

$164.77(\mathrm{C}(3)) ; 106.62(\mathrm{C}(2)) ; 65.92$ (C(5)); 42.59 (C(4)); 18.17 (C(9)); 15.13 $(\mathrm{C}(7)) ; 12.17(\mathrm{C}(8)) ; 11.26(\mathrm{C}(1))$

General Procedure II: Aldol Addition of $(Z)-5$ : $(1 R, 2 R, 4 S)-5$-tert-Butyldimethylsilyloxy-2,4dimethyl-1-hydroxy-1-phenyl-3-pentanone (syn,syn-16a)

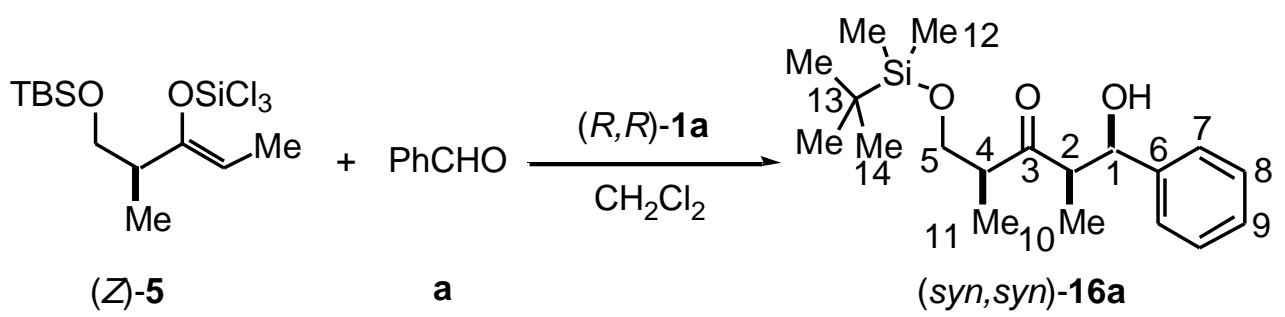

Trichlorosilyl enol ether $(Z)-5(364 \mathrm{mg}, 1.00 \mathrm{mmol})$ was added to a solution of $(R, R)-\mathbf{1 a}$ (37 mg, $0.10 \mathrm{mmol}, 0.10$ equiv) in $1.00 \mathrm{~mL}$ of $\mathrm{CH}_{2} \mathrm{Cl}_{2}$. The reaction mixture was cooled to -78 
${ }^{\circ} \mathrm{C}$ using an acetone/ $\mathrm{CO}_{2}$ bath, and $\mathrm{PhCHO}(102 \mu \mathrm{L}, 1.00 \mathrm{mmol}, 1$ equiv) was added by syringe. After stirring for $6 \mathrm{~h}$, the reaction mixture was quenched by pouring into a vigorously stirred solution of cold, sat. aq. $\mathrm{NaHCO}_{3}$ solution. The resulting slurry was stirred for $1 \mathrm{~h}$ and was filtered through Celite. The layers were separated and the aqueous layer was extracted with 10 $\mathrm{mL}$ of $\mathrm{CH}_{2} \mathrm{Cl}_{2}$. The organic phases were combined, washed with $2 \mathrm{~mL}$ of brine and were dried $\left(\mathrm{Na}_{2} \mathrm{SO}_{4}\right)$, filtered, and concentrated. The crude product was purified by column chromatography (pentane/ether, $\left.3 / 1, \mathrm{SiO}_{2}\right)$ to afford $241 \mathrm{mg}(0.72 \mathrm{mmol}, 72 \%)$ of 16a as a clear, viscous oil.

Data for $(s y n, s y n)-16 a:$

${ }^{1} \underline{\mathrm{H} \text { NMR}}: \quad\left(500 \mathrm{MHz}, \mathrm{CDCl}_{3}\right)$

7.34-7.32 (m, $4 \mathrm{H}, \mathrm{HC}(7), \mathrm{HC}(8)) ; 7.25$ (m, $1 \mathrm{H}, \mathrm{HC}(9)) ; 5.22$ (t, $J=2.4,1 \mathrm{H}$, $\mathrm{HC}(1)) ; 3.75-3.60\left(\mathrm{ABX}, 2 \mathrm{H}, \mathrm{H}_{2} \mathrm{C}(5)\right) ; 3.45$ (d, $\left.J=2.4,1 \mathrm{H}, \mathrm{HO}\right) ; 3.02$ (sext, $J=$ 5.4, $1 \mathrm{H}, \mathrm{HC}(4)) ; 2.97$ (qd, $J=7.1,2.7,1 \mathrm{H}, \mathrm{HC}(2)) ; 1.02$ (d, $J=6.8,3 \mathrm{H}$, $\left.\mathrm{H}_{3} \mathrm{C}(11)\right) ; 1.01$ (d, $\left.J=7.3,3 \mathrm{H}, \mathrm{H}_{3} \mathrm{C}(10)\right) ; 0.89$ (s, 9H, $\left.\mathrm{H}_{3} \mathrm{C}(14)\right) ; 0.06$ (d, $J=3.2$, $\left.6 \mathrm{H}, \mathrm{H}_{3} \mathrm{C}(12)\right)$

${ }^{13}$ C NMR: $\quad\left(126 \mathrm{MHz}, \mathrm{CDCl}_{3}\right)$

$219.19(\mathrm{C}(3)) ; 141.65(\mathrm{C}(6)) ; 128.14(\mathrm{C}(8)) ; 127.06(\mathrm{C}(9)) ; 125.83(\mathrm{C}(7)) ; 71.86$ (C(5)); 66.27 (C(1)); 52.79 (C(2)); 47.31 (C(4)); 25.85 (C(14)); 18.25 (C(13)); $13.17(\mathrm{C}(11)) ; 8.83(\mathrm{C}(10)) ;-5.57(\mathrm{C}(12))$

IR: (neat) 3469 (br, w); 2954 (m); 2931 (m); 2858 (m); 1702 (m); 1462 (m); 1389 (w); 1255 (m); 1097 (m)

MS: (FI)

$336.2\left(\mathrm{M}^{+}, 40\right) ; 279.1$ (100); 265.1 (24); 230.2 (12); 173.1 (6); 159.1 (3); 106.1

Opt. Rot.: $\quad[\alpha]_{\mathrm{D}}^{24}-9.16(\mathrm{c}=2.0, \mathrm{EtOH})$

TLC: $\quad R_{f} 0.33$ (pentane/ether, 3/1) [silica gel, p-anisaldehyde]

SFC: $\quad t_{\mathrm{R}} 1.834 \mathrm{~min}(\mathrm{AD}, 150 \mathrm{bar}, 3 \mathrm{~mL} / \mathrm{min}, 5 \% \mathrm{MeOH})$ 
Analysis: $\quad \mathrm{C}_{19} \mathrm{H}_{32} \mathrm{O}_{3} \mathrm{Si}(336.54)$

$\begin{array}{lll}\text { Calcd: } & \text { C, 67.81; } & \text { H, 9.58\% } \\ \text { Found: } & \text { C, 67.74; } & \text { H, } 9.69 \%\end{array}$

\section{$(1 S, 2 S, 4 S)-5$-tert-Butyldimethylsilyloxy-2,4-dimethyl-1-hydroxy-1-phenyl-3-pentanone} (syn,anti-16a)

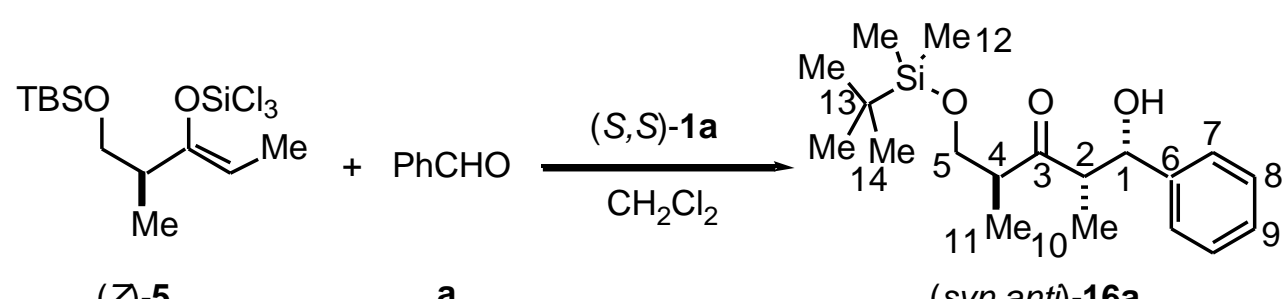

Following General Procedure II from (Z)-5 (364 mg, 1.00 mmol), PhCHO (102 $\mu \mathrm{L}, 1.00$ mmol, 1.0 equiv) and ( $S$ )-1a (37 mg, $0.10 \mathrm{mmol}, 0.1$ equiv) in $1 \mathrm{~mL}$ of $\mathrm{CH}_{2} \mathrm{Cl}_{2}$, was obtained $241 \mathrm{mg}(0.72 \mathrm{mmol}, 72 \%)$ of $\mathbf{1 6 a}$ as a clear, colorless, viscous oil after column chromatography (pentane/ether, 3/1, $\mathrm{SiO}_{2}$ ).

Data for (syn,anti)-16a:

1․ NMR: $\quad\left(500 \mathrm{MHz}, \mathrm{CDCl}_{3}\right)$

7.34 (d, $J=4.2,4 \mathrm{H}, \mathrm{HC}(7), \mathrm{HC}(8)) ; 7.25$ (m, 1H, HC(9)); 5.05 (t, $J=3.2,1 \mathrm{H}$, $\mathrm{HC}(1)) ; 3.81-3.53\left(\mathrm{ABX}, 2 \mathrm{H}, \mathrm{H}_{2} \mathrm{C}(5)\right) ; 3.23$ (d, $J=2.2,1 \mathrm{H}, \mathrm{HO}$ ); 2.95 (qd, $J=$ 6.9, 2.9, 1H, $\mathrm{HC}(2)) ; 2.89$ (sext, $J=5.4,1 \mathrm{H}, \mathrm{HC}(4)) ; 1.04$ (d, $J=7.1,3 \mathrm{H}$, $\left.\mathrm{H}_{3} \mathrm{C}(11)\right) ; 0.89$ (d, $\left.J=6.6,3 \mathrm{H}, \mathrm{H}_{3} \mathrm{C}(10)\right) ; 0.87$ (s, 9H, $\mathrm{H}_{3} \mathrm{C}(14)$ ); 0.03 (d, $J=6.4$, $\left.6 \mathrm{H}, \mathrm{H}_{3} \mathrm{C}(12)\right)$

${ }^{13}$ C NMR: $\quad\left(126 \mathrm{MHz}, \mathrm{CDCl}_{3}\right)$

218.07 (C(3)); $142.06(\mathrm{C}(6)) ; 128.19(\mathrm{C}(8)) ; 127.31(\mathrm{C}(9)) ; 126.05(\mathrm{C}(7)) ; 73.51$ (C(5)); 65.29 (C(1)); $52.76(\mathrm{C}(2)) ; 48.03$ (C(4)); 25.79 (C(14)); 18.19 (C(13)); $12.92(\mathrm{C}(11)) ; 9.89(\mathrm{C}(10)) ;-5.64(\mathrm{C}(12))$

IR: (neat)

3442 (br, m); 3064 (w); 3032 (w); 2956 (s); 2932 (s); 2859 (s); 1706 (s); 1462 (m); $1388(\mathrm{~m}) ; 1361$ (m); 1257 (s); 1099 (s); 1001 (s) 
MS: (FI)

$336.2\left(\mathrm{M}^{+}, 58\right) ; 279.1$ (100); 230.2 (13); 173.1 (5);106.1 (6)

Opt. Rot.: $\quad[\alpha]_{\mathrm{D}}^{24}+49.34(\mathrm{c}=3.8, \mathrm{EtOH})$

TLC: $\quad R_{f} 0.33$ (pentane/ether, 3/1) [silica gel, $p$-anisaldehyde]

SFC: $\quad t_{\mathrm{R}} 2.278 \mathrm{~min}(\mathrm{AD}, 150 \mathrm{bar}, 3 \mathrm{~mL} / \mathrm{min}, 5 \% \mathrm{MeOH})$

Analysis: $\quad \mathrm{C}_{19} \mathrm{H}_{32} \mathrm{O}_{3} \mathrm{Si}(336.54)$

Calcd: $\quad$ C, 67.81; $\quad \mathrm{H}, 9.58 \%$

Found: $\quad$ C, $67.74 ; \quad H, 9.73 \%$

$(1 R, 2 R, 4 S)-5-T r i i s o p r o p y l s i l y l o x y-2,4-d i m e t h y l-1-h y d r o x y-1-p h e n y l-3-p e n t a n o n e ~(s y n, s y n-$ 17a)

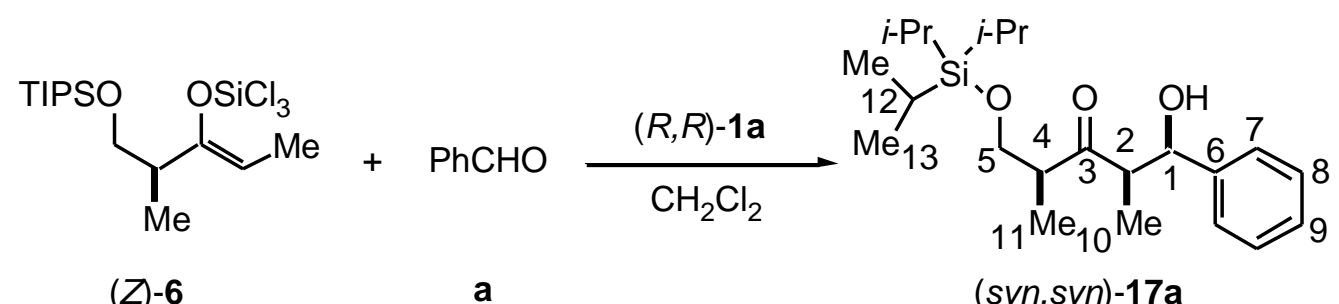

Following General Procedure II from (Z)-6 (479 mg, $1.00 \mathrm{mmol}), \mathrm{PhCHO}(102 \mu \mathrm{L}, 1.00$ mmol, 1.0 equiv) and $(R, R)-\mathbf{1 a}\left(37 \mathrm{mg}, 0.10 \mathrm{mmol}, 0.1\right.$ equiv) in $1 \mathrm{~mL}$ of $\mathrm{CH}_{2} \mathrm{Cl}_{2}$ for $8 \mathrm{~h}$, was obtained $319 \mathrm{mg}(0.84 \mathrm{mmol}, 84 \%)$ of $\mathbf{1 7 a}$ as a clear, colorless, viscous oil after column chromatography (pentane/ether, $5 / 1, \mathrm{SiO}_{2}$ ).

Data for $(s y n, s y n)-\mathbf{1 7 a}$ :

${ }^{1} \underline{\mathrm{H} N M R}: \quad\left(500 \mathrm{MHz}, \mathrm{CDCl}_{3}\right)$

7.35-7.31 (m, $4 \mathrm{H}, \mathrm{HC}(7), \mathrm{HC}(8)) ; 7.25$ (m, $1 \mathrm{H}, \mathrm{HC}(9)) ; 5.23$ (t, $J=2.5,1 \mathrm{H}$, $\mathrm{HC}(1)$ ); 3.85-3.67 (ABX, $\left.2 \mathrm{H}, \mathrm{H}_{2} \mathrm{C}(5)\right)$; 3.47 (d, $\left.J=2.2,1 \mathrm{H}, \mathrm{HO}\right) ; 3.01$ (sext, $J=$ 5.4, $1 \mathrm{H}, \mathrm{HC}(4)) ; 2.98(\mathrm{qd}, J=7.3,2.7,1 \mathrm{H}, \mathrm{HC}(2)) ; 1.14-1.02(\mathrm{~m}, 27 \mathrm{H}$, $\left.\mathrm{H}_{3} \mathrm{C}(11), \mathrm{H}_{3} \mathrm{C}(10), \mathrm{H}_{3} \mathrm{C}(13), \mathrm{HC}(12)\right)$ 
${ }^{13} \underline{\mathrm{C} \mathrm{NMR}}: \quad\left(126 \mathrm{MHz}, \mathrm{CDCl}_{3}\right)$

$219.76(\mathrm{C}(3)) ; 141.86(\mathrm{C}(6)) ; 128.38(\mathrm{C}(8)) ; 127.29(\mathrm{C}(9)) ; 126.06(\mathrm{C}(7)) ; 72.08$ $(\mathrm{C}(5)) ; 66.96$ (C(1)); 53.10 (C(4)); 47.72 (C(2)); 18.18 (C(13)); 13.54 (C(11)); $12.04(\mathrm{C}(12)) ; 9.05(\mathrm{C}(10))$

IR: (neat)

3467 (br, m); 2942 (s); 2891 (s); 2868 (s); 1703 (s); 1462 (s); 1385 (m); 1101 (s); $1070(\mathrm{~m}) ; 997$ (s)

MS: $\quad(\mathrm{FI})$

$379.4\left(\mathrm{M}^{+}, 6\right) ; 337.3(7) ; 336.3(26) ; 335.3(100)$

Opt. Rot.: $\quad[\alpha]_{\mathrm{D}}^{24}-10.84(\mathrm{c}=1.01, \mathrm{EtOH})$

TLC: $\quad R_{f} 0.23$ (pentane/ether, 5/1) [silica gel, $p$-anisaldehyde]

SFC: $\quad t_{\mathrm{R}} 2.220 \mathrm{~min}(\mathrm{AD}, 150 \mathrm{bar}, 3 \mathrm{~mL} / \mathrm{min}, 5 \% \mathrm{MeOH})$

Analysis: $\quad \mathrm{C}_{22} \mathrm{H}_{38} \mathrm{O}_{3} \mathrm{Si}(378.62)$

Calcd: $\quad$ C, 69.79; H, $10.12 \%$

Found: $\quad \mathrm{C}, 69.53 ; \quad \mathrm{H}, 10.24 \%$

$(1 S, 2 S, 4 S)-1-T r i i s o p r o p y l s i l y l o x y-2,4-d i m e t h y l-5-h y d r o x y-5-p h e n y l-3-p e n t a n o n e \quad$ (syn,anti17a)

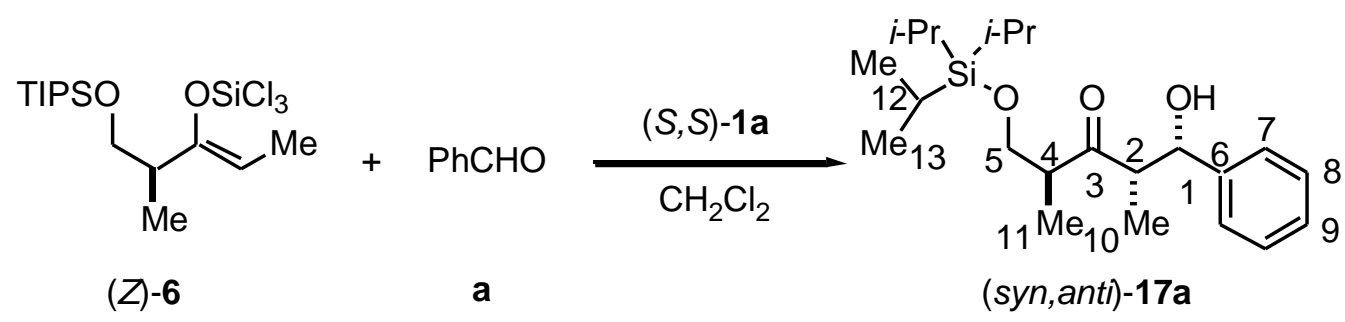

Following General Procedure II from (Z)-6 (479 mg, $1.00 \mathrm{mmol})$, PhCHO (102 $\mu \mathrm{L}, 1.00$ mmol, 1.0 equiv) and $(S, S)-\mathbf{1 a}\left(37 \mathrm{mg}, 0.10 \mathrm{mmol}, 0.1\right.$ equiv) in $1 \mathrm{~mL}$ of $\mathrm{CH}_{2} \mathrm{Cl}_{2}$ for $8 \mathrm{~h}$, was obtained $310 \mathrm{mg}(0.82 \mathrm{mmol}, 82 \%)$ of $\mathbf{1 7 a}$ as a clear, colorless, viscous oil after column chromatography (pentane/ether, $5 / 1, \mathrm{SiO}_{2}$ ).

Data for (syn,anti)-17a:

mp: $\quad 22{ }^{\circ} \mathrm{C}$ (hexane) 
${ }^{1} \underline{\mathrm{H} \text { NMR: }} \quad\left(500 \mathrm{MHz}, \mathrm{CDCl}_{3}\right)$

7.34-7.25 (m, $5 \mathrm{H}, \mathrm{HC}(7), \mathrm{HC}(8), \mathrm{HC}(9)) ; 5.06$ (dd, $J=3.9,2.4,1 \mathrm{H}, \mathrm{HC}(1))$; 3.91-3.61 (ABX, $\left.2 \mathrm{H}, \mathrm{H}_{2} \mathrm{C}(5)\right) ; 3.18(\mathrm{~d}, J=2.2,1 \mathrm{H}, \mathrm{HO}) ; 2.99$ (qd, $J=7.1,4.1$, $1 \mathrm{H}, \mathrm{HC}(2)$ ); 2.89 (sext, $J=5.1,1 \mathrm{H}, \mathrm{HC}(4)) ; 1.08$ (d, $J=7.1,3 \mathrm{H}, \mathrm{H}_{3} \mathrm{C}(11)$ ); $1.09-1.02$ (m, $\left.21 \mathrm{H}, \mathrm{H}_{3} \mathrm{C}(13), \mathrm{HC}(12)\right) ; 0.91$ (d, $\left.J=7.1,3 \mathrm{H}, \mathrm{H}_{3} \mathrm{C}(10)\right)$

${ }^{13}$ C NMR: $\quad\left(126 \mathrm{MHz}, \mathrm{CDCl}_{3}\right)$

$218.30(\mathrm{C}(3)) ; 141.93(\mathrm{C}(6)) ; 128.23(\mathrm{C}(8)) ; 127.33(\mathrm{C}(9)) ; 126.01(\mathrm{C}(7)) ; 73.35$ $(\mathrm{C}(5))$; 65.64 (C(1)); 52.92 (C(4)); 48.28 (C(2)); 17.92 (C(13)); 13.17 (C(11)); $11.83(\mathrm{C}(12)) ; 9.62(\mathrm{C}(10))$

IR: (neat)

3367 (br, m); 2943 (s); 2866 (s); 1707 (s); 1462 (s); 1285 (m); 1265 (m); 1248 (w); 1132 (m); 1097 (s); 1065 (s); 1003 (s)

MS: $\quad(\mathrm{FI})$

$379.2\left(\mathrm{M}^{+}, 5\right) ; 337.2$ (7); 336.2 (25); 335.2 (100); 272.1 (10); 229.1 (14); 157.4 (5); 156.9 (7); 156.7 (7); 126.6 (7); 156.5 (6); 156.3 (7); 106.0 (6)

Opt. Rot.: $\quad[\alpha]_{\mathrm{D}}^{24}+92.03(\mathrm{c}=0.59$, EtOH)

TLC: $\quad R_{f} 0.18$ (pentane/ether, 5/1) [silica gel, $p$-anisaldehyde]

SFC: $\quad t_{\mathrm{R}} 2.900 \mathrm{~min}(\mathrm{AD}, 150 \mathrm{bar}, 3 \mathrm{~mL} / \mathrm{min}, 5 \% \mathrm{MeOH})$

Analysis: $\quad \mathrm{C}_{22} \mathrm{H}_{38} \mathrm{O}_{3} \mathrm{Si}(378.62)$

Calcd: $\quad$ C, 69.79; H, 10.12\%

Found: $\quad$ C, $69.67 ; \quad H, 9.95 \%$ 


\section{$(1 R, 2 R, 4 S)-5$-Triisopropylsilyloxy-2,4-dimethyl-1-hydroxy-1-(1-naphthyl)-3-pentanone} (syn,syn-17b)

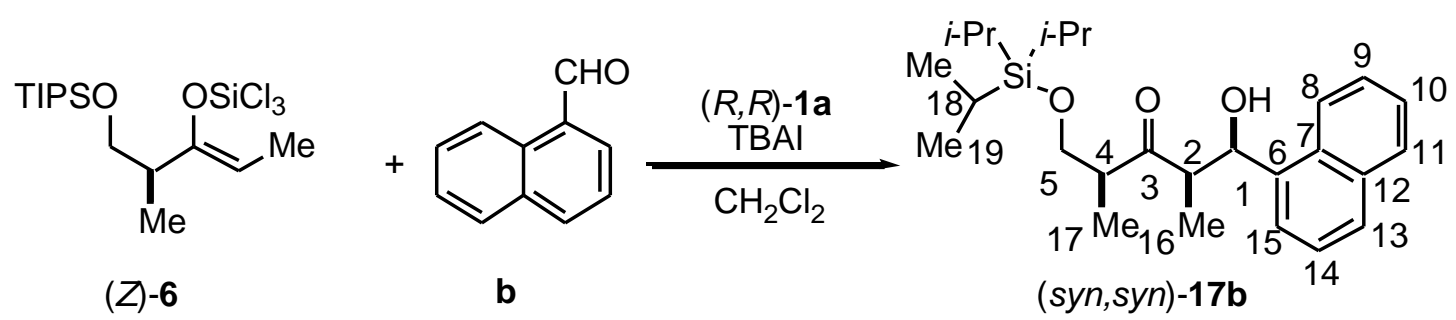

Following General Procedure II from (Z)-6 (472 mg, $1.00 \mathrm{mmol})$, 1-naphthaldehyde (136 $\mu \mathrm{L}, 1.00 \mathrm{mmol}, 1.0$ equiv), $(R, R)-\mathbf{1 a}(37 \mathrm{mg}, 0.10 \mathrm{mmol}, 0.1$ equiv) and tetrabutylammonium iodide (TBAI) $\left(73.9 \mathrm{mg}, 0.20 \mathrm{mmol}, 0.2\right.$ equiv) in $1 \mathrm{~mL}$ of $\mathrm{CH}_{2} \mathrm{Cl}_{2}$ for $8 \mathrm{~h}$, was obtained $313 \mathrm{mg}$ $(0.71 \mathrm{mmol}, 71 \%)$ of $\mathbf{1 7 b}$ as a clear, colorless, viscous oil after column chromatography (pentane/ether, 5/1, $\mathrm{SiO}_{2}$ ). Analytically pure material was obtained by recrystallization from pentane.

Data for $(s y n, s y n)-\mathbf{1 7 b}$ :

mp: $\quad 50-52{ }^{\circ} \mathrm{C}$ (pentane)

1슬 $\quad\left(500 \mathrm{MHz}, \mathrm{CDCl}_{3}\right)$

$7.89-7.88(\mathrm{~m}, 2 \mathrm{H}, \mathrm{HC}(8), \mathrm{HC}(11)) ; 7.77(\mathrm{~d}, J=10,1 \mathrm{H}, \mathrm{HC}(13)) ; 7.76(\mathrm{~d}, J=$ 10, $1 \mathrm{H}, \mathrm{HC}(15)) ; 7.51$ (t, $J=7.4,1 \mathrm{H}, \mathrm{HC}(9)) ; 7.55-7.42$ (m, $2 \mathrm{H}, \mathrm{HC}(14)$, $\mathrm{HC}(10)$ ); 6.03 (br s, $1 \mathrm{H}, \mathrm{HC}(1))$; 3.95-3.72 (ABX, 2 H, $\left.\mathrm{H}_{2} \mathrm{C}(5)\right)$ ) 3.66 (d, J = 2.4, $1 \mathrm{H}, \mathrm{HO}) ; 3.21(\mathrm{qd}, J=7.1,2.5,1 \mathrm{H}, \mathrm{HC}(2)) ; 3.10(\operatorname{sextd}, J=6.8,1.7,1 \mathrm{H}$, $\mathrm{HC}(4)) ; 1.13$ - 1.00 (m, $\left.27 \mathrm{H}, \mathrm{H}_{3} \mathrm{C}(13), \mathrm{H}_{3} \mathrm{C}(16), \mathrm{HC}(18), \mathrm{H}_{3} \mathrm{C}(19)\right)$

${ }^{13} \mathrm{C} \mathrm{NMR}: \quad\left(126 \mathrm{MHz}, \mathrm{CDCl}_{3}\right)$

219.46 (C(3)); 136.77 (C(6)); 133.97 (C(12)); 129.94 (C(7)); 129.45 (C(11)); 127.97 (C(13)); 126.23 (C(10)); 125.72 (C(9)); 125.56 (C(14)); 124.79 (C(15)); 122.62 (C(8)); 68.74 (C(1)); 66.71 (C(5); 51.11 (C(4)); 48.15 (C(2)); 18.26 (C(19)); 13.97 (C(17)); 12.19 (C(18)); 9.61 (C(16))

IR: $\quad\left(\mathrm{CH}_{2} \mathrm{Cl}_{2}\right)$

3469 (br, m); 3052 (w); 2943 (s); 2867 (s); 1704 (m); 1598 (w); 1511 (w); 1462 (m); $1383(\mathrm{w}) ; 1253(\mathrm{w}) ; 1101(\mathrm{~m}) ; 1068$ (m) 
MS: $\quad(\mathrm{FI})$

429.2 (14); $428.2\left(\mathrm{M}^{+}, 37\right) ; 385.2$ (12); 336.2 (25); 272.1 (14); 230.0 (17); 229.1 (100); 155.9 (69)

Opt. Rot.: $\quad[\alpha]_{\mathrm{D}}^{24}+2.1(\mathrm{c}=0.48, \mathrm{EtOH})$

TLC: $\quad R_{f} 0.30$ (pentane/ether, 4/1) [silica gel, short UV]

SFC: $\quad t_{\mathrm{R}} 5.555 \mathrm{~min}(\mathrm{OD}, 150 \mathrm{bar}, 3 \mathrm{~mL} / \mathrm{min}, 15 \% \mathrm{MeOH})$

Analysis: $\quad \mathrm{C}_{26} \mathrm{H}_{40} \mathrm{O}_{3} \mathrm{Si}(428.68)$

Calcd: $\quad$ C, $72.85 ; \quad H, 9.41 \%$

Found: $\quad$ C, $72.52 ; \quad H, 9.40 \%$

\section{(1S,2S,4S)-5-Triisopropylsilyloxy-2,4-dimethyl-1-hydroxy-1-(1-naphthyl)-3-pentanone} (syn,anti-17b)

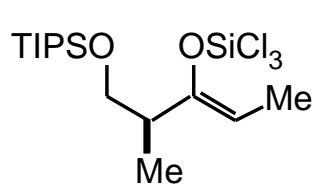

$(Z)-6$

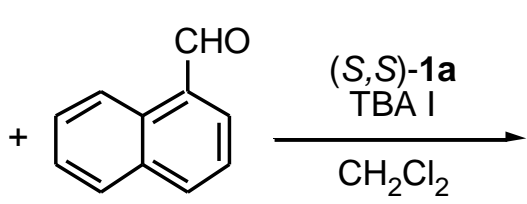

b

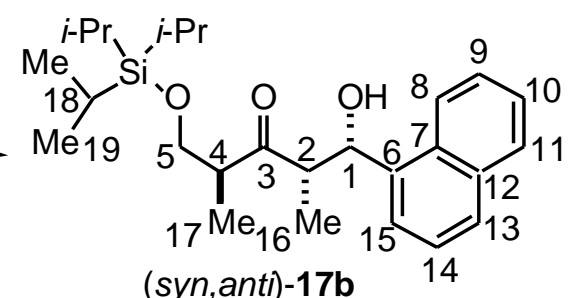

(syn,anti)-17b

Following General Procedure II from (Z)-6 (501 mg, $1.00 \mathrm{mmol}, 19 \%$ bisenoxysilane), 1naphthaldehyde (136 $\mu \mathrm{L}, 1.00 \mathrm{mmol}, 1.0$ equiv), $(S, S)-1 \mathbf{a}(37 \mathrm{mg}, 0.10 \mathrm{mmol}, 0.1 \mathrm{equiv})$ and TBAI (73.9 mg, $0.20 \mathrm{mmol}, 0.2$ equiv) in $1 \mathrm{~mL}$ of $\mathrm{CH}_{2} \mathrm{Cl}_{2}$ for $8 \mathrm{~h}$, was obtained $335 \mathrm{mg}(0.78$ mmol, $78 \%$ ) of $\mathbf{1 7 b}$ as a clear, colorless, viscous oil after column chromatography (pentane/ether, 5/1, $\mathrm{SiO}_{2}$ ).

Data for (syn,anti)-17b:

${ }^{1}$ H NMR: $\quad\left(500 \mathrm{MHz}, \mathrm{CDCl}_{3}\right)$

$7.92(\mathrm{~d}, J=8.0,1 \mathrm{H}, \mathrm{HC}(8)) ; 7.89(\mathrm{~d}, J=7.4,1 \mathrm{H}, \mathrm{HC}(11)) ; 7.78(\mathrm{~d}, J=8.0,1$ H, HC(13)); 7.74 (d, $J=7.3,1$ H, HC(15)); 7.53 - 7.47 (m, 3 H, HC(10), HC(14), $\mathrm{C}(9)$ ); 5.90 (br d, $J=2.2,1 \mathrm{H}, \mathrm{HC}(1)) ; 3.99-3.66\left(\mathrm{ABX}, 2 \mathrm{H}, \mathrm{H}_{2} \mathrm{C}(5)\right) ; 3.57$ (d, $J$ $=2.2,1 \mathrm{H}, \mathrm{HO}) ; 3.20(\mathrm{qd}, J=7.0,1.7,1 \mathrm{H}, \mathrm{HC}(2)) ; 3.00(\operatorname{sextd}, J=7.1,1.7,1 \mathrm{H}$, $\mathrm{HC}(4)) ; 1.12-0.98$ (m, $\left.27 \mathrm{H}, \mathrm{H}_{3} \mathrm{C}(17), \mathrm{H}_{3} \mathrm{C}(19), \mathrm{HC}(18), \mathrm{H}_{3} \mathrm{C}(16)\right)$ 
${ }^{13} \underline{\mathrm{C} \mathrm{NMR}}: \quad\left(126 \mathrm{MHz}, \mathrm{CDCl}_{3}\right)$

$219.01(\mathrm{C}(3))$; $136.66(\mathrm{C}(6)) ; 133.70$ (C(12)); $129.65(\mathrm{C}(7)) ; 129.14(\mathrm{C}(11))$; 127.78 (C(13)); 126.06 (C(10)); 125.37 (C(9)); 125.30 (C(14)); 124.35 (C(15)); $122.41(\mathrm{C}(8)) ; 69.66$ (C(1)); 65.73 (C(5)); 50.11 (C(4)); 48.07 (C(2)); 17.92 (C(19)); $15.27(\mathrm{C}(17)) ; 11.86(\mathrm{C}(18)) ; 9.30(\mathrm{C}(16))$

IR: (neat)

3470 (br, m); 3052 (w); 2943 (s); 2892 (m); 1703 (m); 1598 (w); 1511 (w); 1462 (m); $1383(\mathrm{~m}) ; 1253(\mathrm{w}) ; 1102(\mathrm{~s}) ; 1068$ (m)

MS: $\quad(\mathrm{FI})$

430.2 (13); 429.2 (26); $428.2\left(\mathrm{M}^{+}, 48\right) ; 385.2$ (58); 346.6 (10); 273.2 (13); 272.2 (46); 230.1 (13); 229.1 (92); 156.0 (100)

Opt. Rot.: $\quad[\alpha]_{\mathrm{D}}^{24}+47.9(\mathrm{c}=0.97$, EtOH $)$

TLC: $\quad R_{f} 0.26$ (pentane/ether, 5/1) [silica gel, short UV]

SFC: $\quad t_{\mathrm{R}} 3.44 \mathrm{~min}(\mathrm{OD}, 150 \mathrm{bar}, 3 \mathrm{~mL} / \mathrm{min}, 15 \% \mathrm{MeOH})$

Analysis: $\quad \mathrm{C}_{26} \mathrm{H}_{40} \mathrm{O}_{3} \mathrm{Si}(428.68)$

Calcd: $\quad$ C, $72.85 ; \quad H, 9.41 \%$

Found: $\quad$ C, $72.47 ; \quad$ H, $9.29 \%$

\section{(2S,4R,5R)-1-Triisopropylsilyloxy-2,4-dimethyl-5-hydroxy-7-phenyl-6-hepten-3-one} $(\text { syn,syn-17c) })^{9}$

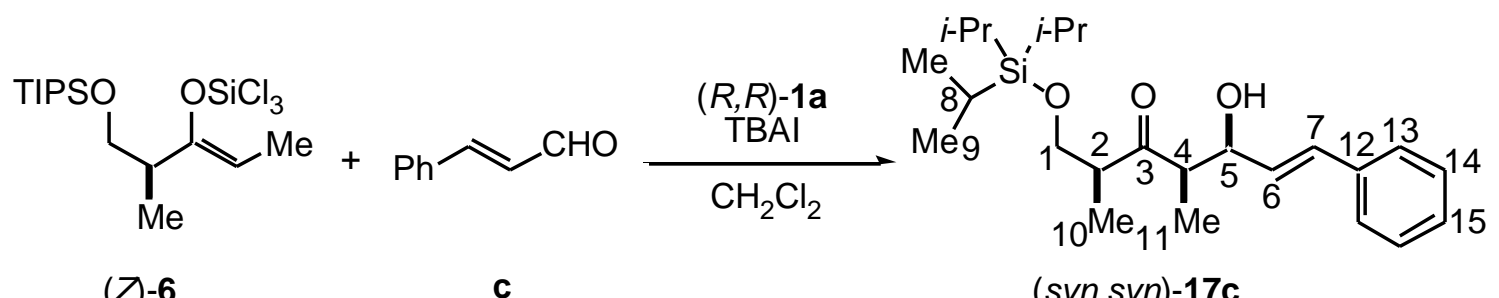

Following General Procedure II from (Z)-6 (872 mg, $2.00 \mathrm{mmol})$, cinnamaldehyde (252 $\mu \mathrm{L}, 2.00 \mathrm{mmol}, 1.0$ equiv), $(R, R)-\mathbf{1 a}(74 \mathrm{mg}, 0.20 \mathrm{mmol}, 0.1$ equiv) and TBAI (148 mg, 0.40 mmol, 0.2 equiv) in $2 \mathrm{~mL}$ of $\mathrm{CH}_{2} \mathrm{Cl}_{2}$ for $10 \mathrm{~h}$, was obtained $713 \mathrm{mg}(1.76 \mathrm{mmol}, 88 \%)$ of $\mathbf{1 7 c}$ as a clear, colorless, viscous oil after column chromatography (pentane/ether, 3/1, $\mathrm{SiO}_{2}$ ). 
Data for $($ syn, syn)-17c:

1슬 $: \quad\left(500 \mathrm{MHz}, \mathrm{CDCl}_{3}\right)$

$7.38(\mathrm{~d}, J=7.1,2 \mathrm{H}, \mathrm{HC}(13)) ; 7.31(\mathrm{t}, J=7.5,2 \mathrm{H}, \mathrm{HC}(14)) ; 7.24(\mathrm{t}, J=7.4,1$ H, HC(15)); 6.64 (dd, $J=15.8,0.9,1 \mathrm{H}, \mathrm{HC}(7)) ; 6.13$ (dd, $J=15.8,5.6,1 \mathrm{H}$, $\mathrm{HC}(6))$; 4.75 (m, $1 \mathrm{H}, \mathrm{HC}(5))$; 3.88-3.70 (ABX, $\left.2 \mathrm{H}, \mathrm{H}_{2} \mathrm{C}(1)\right) ; 3.30$ (d, $J=2.9,1$ H, HO); 3.09 (sext, $J=5.4,1 \mathrm{H}, \mathrm{HC}(2)) ; 2.95$ (qd, $J=7.3,2.7,1 \mathrm{H}, \mathrm{HC}(4)) ; 1.17$ $\left(\mathrm{d}, J=7.4,3 \mathrm{H}, \mathrm{H}_{3} \mathrm{C}(11)\right) ; 1.11-1.02$ (m, $\left.24 \mathrm{H}, \mathrm{H}_{3} \mathrm{C}(9), \mathrm{H}_{3} \mathrm{C}(10), \mathrm{HC}(8)\right)$

${ }^{13}$ C NMR: $\quad\left(126 \mathrm{MHz}, \mathrm{CDCl}_{3}\right)$

219.39 (C(3)); 137.07 (C(12)); 130.91 (C(6)); 129.19 (C(7)); $128.76(\mathrm{C}(14))$; 127.75 (C(15)); $126.64(\mathrm{C}(13)) ; 71.48$ (C(1)); 67.19 (C(5)); 51.51 (C(4)); 47.61 $(\mathrm{C}(2)) ; 18.15(\mathrm{C}(9)) ; 13.40(\mathrm{C}(10)) ; 12.01(\mathrm{C}(8)) ; 9.64(\mathrm{C}(11))$

IR: (neat)

3436 (br, m); 3061 (w); 3027 (w); 2943 (s); 2892 (m); 2867 (m); 1703 (m); 1600 (w); 1495(w); 1462 (m); $1384(\mathrm{w}) ; 1368$ (w); 1249 (w); $1102(\mathrm{~m}) ; 1069$ (m)

$\underline{\mathrm{MS}}: \quad(\mathrm{FI})$

406.2 (9); 405.2 (34); $404.2\left(\mathrm{M}^{+}, 100\right) ; 335.2$ (9); 272.1 (8); 229.1 (24); 132.0 (48)

Opt. Rot.: $\quad[\alpha]_{\mathrm{D}}^{24}+20.0\left(\mathrm{c}=0.73, \mathrm{CH}_{2} \mathrm{Cl}_{2}\right)$

$[\alpha]_{\mathrm{D}}^{24}-47.8(\mathrm{c}=0.73, \mathrm{EtOH})$

TLC: $\quad R_{f} 0.12$ (pentane/ether, 3/1) [silica gel, short UV]

SFC: $\quad t_{\mathrm{R}} 3.348 \mathrm{~min}(\mathrm{OD}, 150 \mathrm{bar}, 3 \mathrm{~mL} / \mathrm{min}, 7 \% \mathrm{MeOH})$

Analysis: $\quad \mathrm{C}_{24} \mathrm{H}_{40} \mathrm{O}_{3} \mathrm{Si}(404.66)$

Calcd: $\quad$ C, $71.23 ; \quad \mathrm{H}, 9.96 \%$

Found: $\quad \mathrm{C}, 71.27 ; \quad \mathrm{H}, 10.08 \%$ 


\section{(2S,4S,5S)-1-Triisopropylsilyloxy-2,4-dimethyl-5-hydroxy-7-phenyl-6-hepten-3-one} (syn,anti-17c)

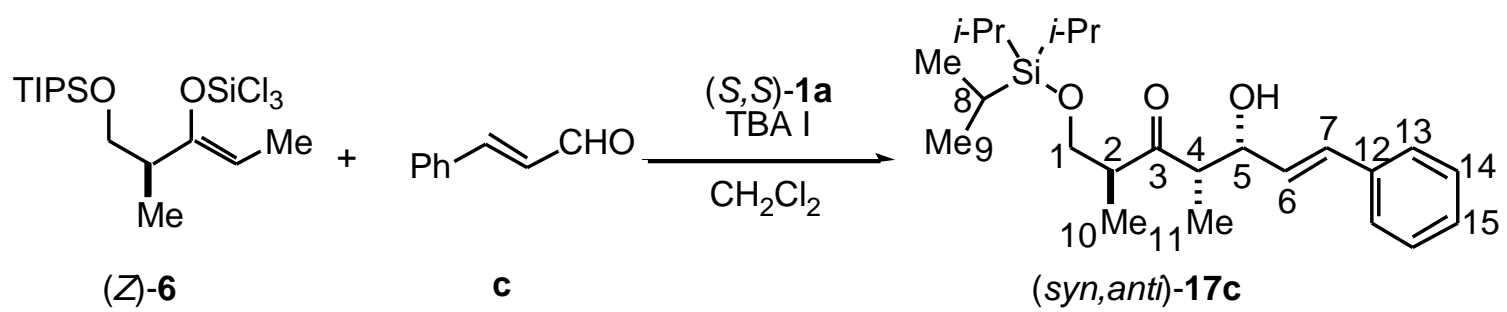

Following General Procedure II from (Z)-6 (436 mg, $1.00 \mathrm{mmol}$ ), cinnamaldehyde (126 $\mu \mathrm{L}, 1.00 \mathrm{mmol}, 1.0$ equiv), $(S, S)-\mathbf{1 a}(37 \mathrm{mg}, 0.10 \mathrm{mmol}, 0.1$ equiv) and TBAI (73.9 mg, 0.20 mmol, 0.2 equiv) in $1 \mathrm{~mL}$ of $\mathrm{CH}_{2} \mathrm{Cl}_{2}$ for $10 \mathrm{~h}$, was obtained $303 \mathrm{mg}(0.75 \mathrm{mmol}, 75 \%)$ of $\mathbf{1 7 c}$ as a clear, colorless, viscous oil after column chromatography (pentane/ether, 3/1, $\mathrm{SiO}_{2}$ ).

Data for (syn,anti)-17c:

1슬 $: \quad\left(500 \mathrm{MHz}, \mathrm{CDCl}_{3}\right)$

7.38 (d, $J=7.6,2 \mathrm{H}, \mathrm{HC}(13)) ; 7.31$ (t, $J=7.6,2 \mathrm{H}, \mathrm{HC}(14)) ; 7.24$ (t, $J=7.8,1$ H, HC(15)); 6.65 (d, $J=16.1,1 \mathrm{H}, \mathrm{HC}(7)) ; 6.13$ (dd, $J=15.8,5.8,1 \mathrm{H}, \mathrm{HC}(6))$; 4.64 (br m, $1 \mathrm{H}, \mathrm{HC}(5)$ ); 3.94-3.65 (ABX, $2 \mathrm{H}, \mathrm{H}_{2} \mathrm{C}(1)$ ); 3.03 (sext, $J=5.4,1 \mathrm{H}$, $\mathrm{HC}(2)) ; 2.99$ (d, $J=2.9,1 \mathrm{H}, \mathrm{HO}) ; 2.92$ (qd, $J=7.3,3.6,1 \mathrm{H}, \mathrm{HC}(4)) ; 1.18$ (d, $J$ $\left.=6.5,3 \mathrm{H}, \mathrm{H}_{3} \mathrm{C}(11)\right) ; 1.16-1.04\left(\mathrm{~m}, 24 \mathrm{H}, \mathrm{H}_{3} \mathrm{C}(9), \mathrm{H}_{3} \mathrm{C}(10), \mathrm{HC}(8)\right)$

${ }^{13}$ C NMR: $\quad\left(101 \mathrm{MHz}, \mathrm{CDCl}_{3}\right)$

$218.13(\mathrm{C}(3))$; $137.07(\mathrm{C}(12)) ; 130.92(\mathrm{C}(6)) ; 129.14(\mathrm{C}(7)) ; 128.54(\mathrm{C}(14))$; 127.60 (C(15)); 126.43 (C(13)); 72.33 (C(1)); 65.85 (C(5)); 50.52 (C(4)); 48.14 $(\mathrm{C}(2)) ; 17.94(\mathrm{C}(9)) ; 15.27$ (C(10)); 11.84 (C(8)); 9.91 (C(11))

IR: (neat) 3430 (br, m); 3083 (w); 3061 (w); 3027 (w); 2943 (s); 2892 (s); 2726 (w); 1706 (s); $1627(\mathrm{w}) ; 1600(\mathrm{w}) ; 1578(\mathrm{w}) ; 1495(\mathrm{~m}) ; 1462$ (s); $1383(\mathrm{~m}) ; 1368(\mathrm{~m}) ; 1250$ (m); $1203(\mathrm{w}) ; 1104(\mathrm{~s}) ; 1068(\mathrm{~s})$

MS: $\quad(\mathrm{FI})$

405.2 (12); $404.2\left(\mathrm{M}^{+}, 34\right) ; 287.1$ (11); 273.1 (15); 269.1 (15); 261.1 (69); 245.1 (30); 229.1 (100); 217.0 (83); 131.9 (17); 106.0 (28) 
Opt. Rot.: $\quad[\alpha]_{\mathrm{D}}^{24}+42.1\left(\mathrm{c}=1.35, \mathrm{CH}_{2} \mathrm{Cl}_{2}\right)$

$$
[\alpha]_{\mathrm{D}}^{24}+109.0(\mathrm{c}=1.25, \mathrm{EtOH})
$$

TLC: $\quad R_{f} 0.23$ (pentane/ether, 3/1) [silica gel, short UV]

SFC: $\quad t_{\mathrm{R}} 6.630 \mathrm{~min}(\mathrm{OD}, 150 \mathrm{bar}, 3 \mathrm{~mL} / \mathrm{min}, 7 \% \mathrm{MeOH})$

Analysis: $\quad \mathrm{C}_{24} \mathrm{H}_{40} \mathrm{O}_{3} \mathrm{Si}(404.66)$
Calcd:
C, 71.23;
H, $9.96 \%$
Found:
C, 71.30;
$\mathrm{H}, 9.79 \%$

(2S,4R,5R)-1-Triisopropylsilyloxy-2,4-dimethyl-5-hydroxy-6-octen-3-one (syn,syn-17d)

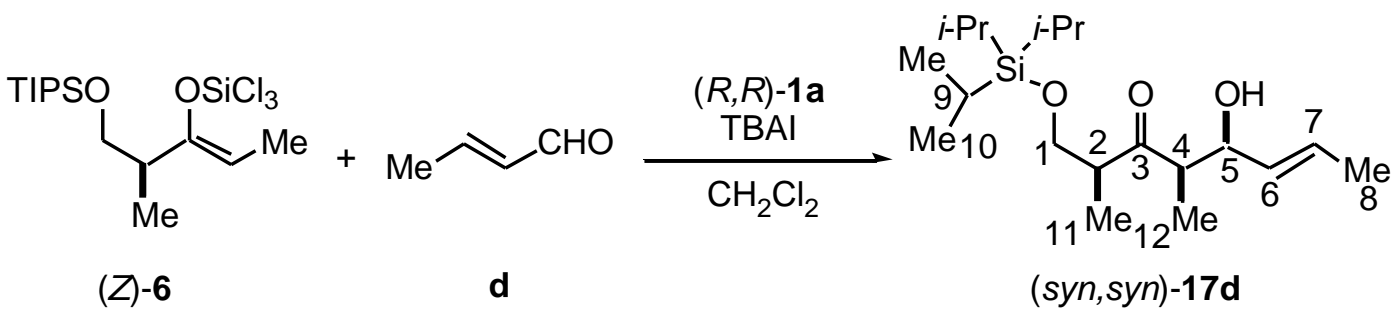

Following General Procedure II from (Z)-6 (472 mg, $1.00 \mathrm{mmol})$, crotonaldehyde (83 $\mu \mathrm{L}$, $1.00 \mathrm{mmol}, 1.0$ equiv), $(R, R)-\mathbf{1 a}(37 \mathrm{mg}, 0.10 \mathrm{mmol}, 0.1$ equiv) and TBAI (73.9 mg, $0.20 \mathrm{mmol}$, 0.2 equiv) in $1 \mathrm{~mL}$ of $\mathrm{CH}_{2} \mathrm{Cl}_{2}$ for $6 \mathrm{~h}$, was obtained $310 \mathrm{mg}(0.90 \mathrm{mmol}, 90 \%)$ of $\mathbf{1 7 d}$ as a clear, colorless, viscous oil after column chromatography (pentane/ether, 3/1, $\mathrm{SiO}_{2}$ ). The diastereomeric ratio of $\mathbf{1 7 d}$ was determined by ${ }^{1} \mathrm{H}$ NMR integration of $\mathrm{HC}(5)$.

Data for $(s y n, s y n)-\mathbf{1 7 d}$ :

${ }^{1} \underline{\mathrm{H} \mathrm{NMR}}: \quad\left(500 \mathrm{MHz}, \mathrm{CDCl}_{3}\right)$

$5.70(\mathrm{dq}, J=16.4,6.0,1 \mathrm{H}, \mathrm{HC}(7)) ; 5.45(\mathrm{dd}, J=6.4,1.5,1 \mathrm{H}, \mathrm{HC}(6)) ; 4.47$ (m, $1 \mathrm{H}, \mathrm{HC}(5)$ ); 3.86-3.67 (ABX, $2 \mathrm{H}, \mathrm{H}_{2} \mathrm{C}(1)$ ); 3.04 (sext, $J=5.4,1 \mathrm{H}, \mathrm{HC}(2)$ ); $3.03(\mathrm{~d}, J=3.2,1 \mathrm{H}, \mathrm{HO}) ; 2.83(\mathrm{qd}, J=7.3,2.9,1 \mathrm{H}, \mathrm{HC}(4)) ; 1.70(\mathrm{dd}, J=5.7$, 1.0, $\left.3 \mathrm{H}, \mathrm{H}_{3} \mathrm{C}(8)\right)$; 1.12 (d, J =7.4, $3 \mathrm{H}, \mathrm{H}_{3} \mathrm{C}(11)$ ); 1.10 - 1.07 (m, $24 \mathrm{H}, \mathrm{H}_{3} \mathrm{C}(10)$, $\left.\mathrm{H}_{3} \mathrm{C}(12), \mathrm{HC}(9)\right)$ 
${ }^{13} \underline{\mathrm{C} \mathrm{NMR}}: \quad\left(126 \mathrm{MHz}, \mathrm{CDCl}_{3}\right)$

219.01 (C(3)); 130.39 (C(7)); 127.32 (C(6); 71.60 (C(1)); 66.61 (C(5)); 51.17 (C(4)); 47.47 (C(2)); 17.81 (C(10)); 17.65 (C(8)); 13.12 (C(11)); 11.72 (C(9)); $9.60(\mathrm{C}(12))$

IR: (neat)

3514 (br, w); 2945 (m); 2868 (m); 1699 (m); 1462 (m); 1383 (w); 1247 (w); 1098 (m); $1068(\mathrm{~m}) ; 1008(\mathrm{~m})$

MS: $\quad(\mathrm{FI})$

$343.2\left(\mathrm{M}^{+}, 5\right) ; 301.1(6) ; 300.2$ (25); 299.2 (100); 272.1 (6); 175.8 (6); 175.6 (9); 175.4 (10); $175.3(8) ; 175.1(6)$

Opt. Rot:: $\quad[\alpha]_{\mathrm{D}}^{24}+5.06(\mathrm{c}=0.23, \mathrm{EtOH})$

TLC: $\quad R_{f} 0.25$ (pentane/ether, 3/1) [silica gel, $p$-anisaldehyde]

Analysis: $\quad \mathrm{C}_{19} \mathrm{H}_{38} \mathrm{O}_{3} \mathrm{Si}(342.59)$

Calcd: $\quad$ C, 66.61; H, $11.18 \%$

Found: $\quad$ C, $66.34 ; \quad H, 11.16 \%$

\section{(2S,4S,5S)-1-Triisopropylsilyloxy-2,4-dimethyl-5-hydroxy-6-octen-3-one (syn,anti-17d)}

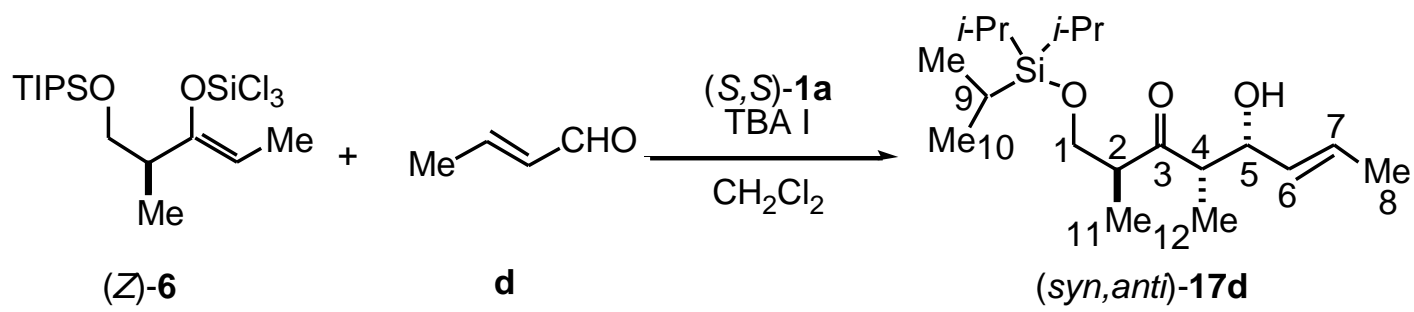

Following General Procedure II from (Z)-6 (472 mg, $1.00 \mathrm{mmol})$, crotonaldehyde (83 $\mu \mathrm{L}$, $1.00 \mathrm{mmol}, 1.0$ equiv), $(S, S)-\mathbf{1 a}(37 \mathrm{mg}, 0.10 \mathrm{mmol}, 0.1$ equiv) and TBAI (73.9 $\mathrm{mg}, 0.20 \mathrm{mmol}$, 0.2 equiv) in $1 \mathrm{~mL}$ of $\mathrm{CH}_{2} \mathrm{Cl}_{2}$ for $6 \mathrm{~h}$, was obtained $293 \mathrm{mg}(0.85 \mathrm{mmol}, 85 \%)$ of $\mathbf{1 7 d}$ as a clear, colorless, viscous oil after column chromatography (pentane/ether, 3/1, $\mathrm{SiO}_{2}$ ). The diastereomeric ratio was determined by ${ }^{1} \mathrm{H}$ NMR integration of $\mathrm{HC}(5)$. 


\section{Data for (syn,anti)-17d:}

${ }^{1}$ H NMR: $\quad\left(500 \mathrm{MHz}, \mathrm{CDCl}_{3}\right)$

$5.72(\mathrm{dq}, J=15.2,6.4,1 \mathrm{H}, \mathrm{HC}(7)) ; 5.46(\mathrm{dd}, J=15.1,6.6,1 \mathrm{H}, \mathrm{HC}(6)) ; 4.36(\mathrm{~m}$, $1 \mathrm{H}, \mathrm{HC}(5)$ ); 3.91-3.63 (ABX, $2 \mathrm{H}, \mathrm{H}_{2} \mathrm{C}(1)$ ); 2.99 (sext, $J=7.6,1 \mathrm{H}, \mathrm{HC}(2)$ ); $2.81(\mathrm{qd}, J=7.0,4.0,1 \mathrm{H}, \mathrm{HC}(4)) ; 2.66$ (d, $J=3.2,1 \mathrm{H}, \mathrm{HO}) ; 1.70$ (dd, $J=7.3$, $\left.0.7,3 \mathrm{H}, \mathrm{H}_{3} \mathrm{C}(8)\right) ; 1.12$ (d, J =7.1, $\left.3 \mathrm{H}, \mathrm{H}_{3} \mathrm{C}(11)\right) ; 1.11-1.00\left(\mathrm{~m}, 24 \mathrm{H}, \mathrm{H}_{3} \mathrm{C}(10)\right.$, $\left.\mathrm{H}_{3} \mathrm{C}(12), \mathrm{HC}(9)\right)$

${ }^{13} \mathrm{C}$ NMR: $\quad\left(126 \mathrm{MHz}, \mathrm{CDCl}_{3}\right)$

$217.83(\mathrm{C}(3)) ; 130.85(\mathrm{C}(7)) ; 127.63(\mathrm{C}(6) ; 72.70(\mathrm{C}(1)) ; 65.82(\mathrm{C}(5)) ; 50.79$ $(\mathrm{C}(4)) ; 48.24(\mathrm{C}(2)) ; 17.84(\mathrm{C}(10)) ; 17.62(\mathrm{C}(8)) ; 13.11(\mathrm{C}(11)) ; 11.75(\mathrm{C}(9))$; $9.60(\mathrm{C}(12))$

IR: (neat)

3468 (br, w); 2944 (m); 2868 (m); 1703 (m); 1462 (m); 1383 (w); 1250 (w); 1104 (m); $1068(\mathrm{~m})$

MS: $\quad(\mathrm{FI})$

$342.2\left(\mathrm{M}^{+}, 1\right) ; 301.2$ (6); 300.1 (23); 299.1 (100); 229.1 (15); 175.4 (7)

Opt. Rot.: $\quad[\alpha]_{\mathrm{D}}^{24}+81.7(\mathrm{c}=0.35$, EtOH $)$

TLC: $\quad R_{f} 0.25$ (pentane/ether, 3/1) [silica gel, $p$-anisaldehyde]

Analysis: $\quad \mathrm{C}_{19} \mathrm{H}_{38} \mathrm{O}_{3} \mathrm{Si}(342.59)$

Calcd: $\quad$ C, 66.61; $\quad \mathrm{H}, 11.18 \%$

Found: $\quad \mathrm{C}, 66.21 ; \quad \mathrm{H}, 11.13 \%$ 
(2S,4R,5R)-1-Triisopropylsilyloxy-2,4,6-trimethyl-5-hydroxy-6-octen-3-one (syn,syn-17e)

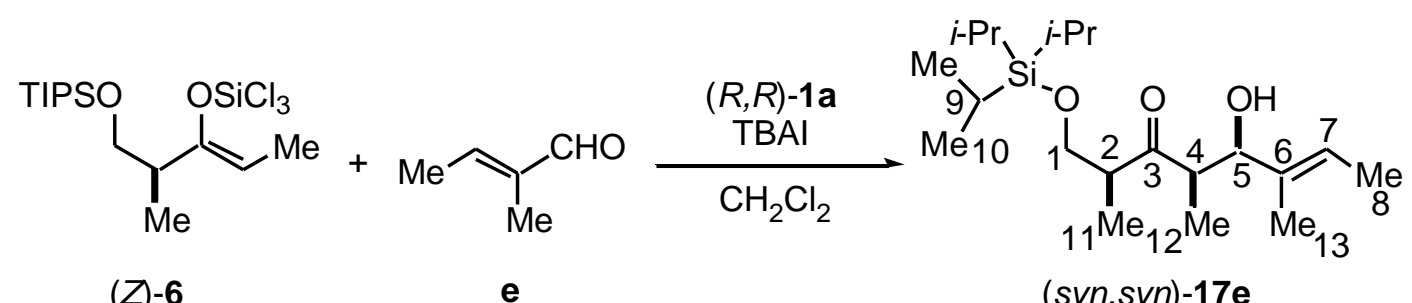

Following General Procedure II from (Z)-6 (472 mg, $1.00 \mathrm{mmol})$, tiglic aldehyde (96 $\mu \mathrm{L}$, $1.00 \mathrm{mmol}, 1.0$ equiv), $(R, R)-\mathbf{1 a}(37 \mathrm{mg}, 0.10 \mathrm{mmol}, 0.1$ equiv) and TBAI (73.9 mg, $0.20 \mathrm{mmol}$, 0.2 equiv) in $1 \mathrm{~mL}$ of $\mathrm{CH}_{2} \mathrm{Cl}_{2}$ for $7 \mathrm{~h}$, was obtained $303 \mathrm{mg}(0.85 \mathrm{mmol}, 85 \%)$ of $\mathbf{1 7 e}$ as a clear, colorless, viscous oil after column chromatography (pentane/ether, 5/1, $\mathrm{SiO}_{2}$ ). The diastereomeric ratio was determined by ${ }^{1} \mathrm{H}$ NMR integration of $\mathrm{HC}(5)$.

Data for $(s y n, s y n)-17 \mathbf{e}$ :

${ }^{1} \underline{\mathrm{H} \mathrm{NMR}}: \quad\left(500 \mathrm{MHz}, \mathrm{CDCl}_{3}\right)$

5.60 (qt, $J=6.8,1.5,1 \mathrm{H}, \mathrm{HC}(7)) ; 4.42$ (m, $1 \mathrm{H}, \mathrm{HC}(5))$; 3.87-3.67 (ABX, $2 \mathrm{H}$, $\left.\mathrm{H}_{2} \mathrm{C}(1)\right) ; 3.11(\mathrm{~d}, J=2.5,1 \mathrm{H}, \mathrm{HO}) ; 3.02$ (sext, $\left.J=6.8,1 \mathrm{H}, \mathrm{HC}(2)\right) ; 2.88(\mathrm{qd}, J=$ 7.3, 2.9, $1 \mathrm{H}, \mathrm{HC}(4)) ; 1.63$ (d, $\left.J=6.3,3 \mathrm{H}, \mathrm{H}_{3} \mathrm{C}(8)\right) ; 1.56$ (s, $\left.3 \mathrm{H}, \mathrm{H}_{3} \mathrm{C}(13)\right) ; 1.10$ - 1.02 (m, $\left.27 \mathrm{H}, \mathrm{H}_{3} \mathrm{C}(11), \mathrm{H}_{3} \mathrm{C}(10), \mathrm{H}_{3} \mathrm{C}(12), \mathrm{HC}(9)\right)$

${ }^{13} \mathrm{C} \mathrm{NMR}: \quad\left(126 \mathrm{MHz}, \mathrm{CDCl}_{3}\right)$

219.22 (C(3)); 133.69 (C(7)); 119.87 (C(6)); 73.77 (C(1)); 66.51 (C(5)); 48.61 (C(4)); 47.53 (C(2)); 17.88 (C(10)); 13.42 (C(8)); 13.27 (C(13)); 12.90 (C(11)); $11.78(\mathrm{C}(9)) ; 8.96(\mathrm{C}(12))$

IR: $\quad\left(\mathrm{CHCl}_{3}\right)$

3584 (br, w); 2945 (m); 2868 (m); 1701 (w); 1461 (w); 1383 (w); 1103 (w)

MS: $\quad(\mathrm{FI})$

$357.3\left(\mathrm{M}^{+}, 7\right) ; 311.2(5) ; 310.3$ (4); 231.2 (5); 230.2 (18); 229.2 (100)

Opt. Rot.: $\quad[\alpha]_{\mathrm{D}}^{24}+5.71(\mathrm{c}=0.20$, EtOH $)$

TLC: $\quad R_{f} 0.20$ (pentane/ether, 5/1) [silica gel, $p$-anisaldehyde] 
Analysis: $\quad \mathrm{C}_{20} \mathrm{H}_{40} \mathrm{O}_{3} \mathrm{Si}(356.62)$

$\begin{array}{lll}\text { Calcd: } & \text { C, } 67.36 ; & \text { H, } 11.31 \% \\ \text { Found: } & \text { C, 66.99; } & \text { H, } 11.28 \%\end{array}$

(2S, 4S, 5S)-1-Triisopropylsilyloxy-2,4,6-trimethyl-5-hydroxy-6-octen-3-one (syn,anti-17e)

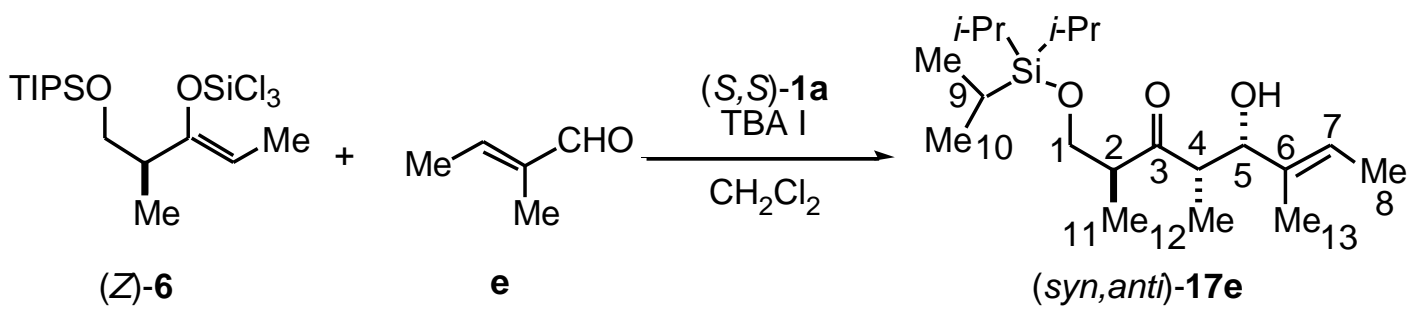

Following General Procedure II from (Z)-6 (472 mg, $1.00 \mathrm{mmol})$, tiglic aldehyde (96 $\mu \mathrm{L}$, $1.00 \mathrm{mmol}, 1.0$ equiv), $(S, S)-\mathbf{1 a}(37 \mathrm{mg}, 0.10 \mathrm{mmol}, 0.1$ equiv) and TBAI (73.9 mg, $0.20 \mathrm{mmol}$, 0.2 equiv) in $1 \mathrm{~mL}$ of $\mathrm{CH}_{2} \mathrm{Cl}_{2}$ for $7 \mathrm{~h}$, was obtained $286 \mathrm{mg}(0.80 \mathrm{mmol}, 80 \%)$ of $\mathbf{1 7 e}$ as a clear, colorless, viscous oil after column chromatography (pentane/ether, 5/1, $\mathrm{SiO}_{2}$ ). The diastereomeric ratio was determined by ${ }^{1} \mathrm{H}$ NMR integration of $\mathrm{HC}(5)$.

Data for (syn,anti)-17e:

${ }^{1}$ H NMR: $\quad\left(500 \mathrm{MHz}, \mathrm{CDCl}_{3}\right)$

5.57 (qt, $J=6.8,1.2,1 \mathrm{H}, \mathrm{HC}(7)) ; 4.36$ (br s, $1 \mathrm{H}, \mathrm{HC}(5)$ ); 3.89-3.63 (ABX, $2 \mathrm{H}$, $\mathrm{H}_{2} \mathrm{C}(1)$ ); 2.99 (sext, $J=5.4,1 \mathrm{H}, \mathrm{HC}(2)$ ); 2.92 (qd, $J=7.1,3.0,1 \mathrm{H}, \mathrm{HC}(4)$ ); 2.64 (d, $J=2.9,1 \mathrm{H}, \mathrm{HO}) ; 1.66$ (d, $J=6.9,3 \mathrm{H}, \mathrm{H}_{3} \mathrm{C}(8)$ ); 1.60 (s, $\left.3 \mathrm{H}, \mathrm{H}_{3} \mathrm{C}(13)\right) ; 1.10$ (m, $27 \mathrm{H}, \mathrm{H}_{3} \mathrm{C}(11), \mathrm{H}_{3} \mathrm{C}(10), \mathrm{H}_{3} \mathrm{C}(12), \mathrm{HC}(9)$ )

${ }^{13}$ C NMR: $\quad\left(126 \mathrm{MHz}, \mathrm{CDCl}_{3}\right)$

$217.92(\mathrm{C}(3)) ; 134.37(\mathrm{C}(6)) ; 120.43(\mathrm{C}(7)) ; 73.79(\mathrm{C}(5)) ; 66.02(\mathrm{C}(1)) ; 48.61$ (C(4)); $48.08(\mathrm{C}(2)) ; 17.86(\mathrm{C}(10)) ; 13.40(\mathrm{C}(8)) ; 13.15$ (C(13)); $12.86(\mathrm{C}(11))$; $11.78(\mathrm{C}(9)) ; 9.49(\mathrm{C}(12))$

IR: (neat)

3468 (br, m); 2944 (m); 2868 (m); 1703 (m); 1463 (m); 1383 (w); 1249 (w); 1102 (m); $1068(\mathrm{w})$ 
MS: $\quad(\mathrm{FI})$

$356.3\left(\mathrm{M}^{+}, 1\right) ; 311.3(5) ; 232.2(5) ; 231.2$ (6); 230.2 (18); 229.2 (100)

Opt. Rot.: $\quad[\alpha]_{\mathrm{D}}^{24}+10.2(\mathrm{c}=0.53$, EtOH $)$

TLC: $\quad R_{f} 0.20$ (pentane/ether, 5/1) [silica gel, $p$-anisaldehyde]

Analysis: $\quad \mathrm{C}_{20} \mathrm{H}_{40} \mathrm{O}_{3} \mathrm{Si}(356.62)$

Calcd: $\quad$ C 67.36; H $11.31 \%$

Found: $\quad$ C $66.96 ; \quad$ H $11.30 \%$

\section{References}

(1) Still, W. C.; Kahn, M.; Mitra, A. J. Org. Chem. 1978, 43, 2923.

(2) Gilman, H.; Cartledge, F. K. J. Organomet. Chem. 1964, 2, 447.

(3) Luke, G. P.; Morris, J. J. Org. Chem. 1995, 60, 3013.

(4) Paterson, I.; Franklin, A. S. Tetrahedron Lett. 1994, 35, 6925.

(5) Denmark, S. E.; Fujimori, S. Synlett 2001, 1024.

(6) Hall, P. L.; Gilchrist, J. H.; Collum, D. B. J. Am. Chem. Soc. 1991, 113, 9571.

(7) Taniguchi, Y.; Inanaga, J.; Yamaguchi, M. Bull. Chem. Soc. Jpn. 1981, 54, 3229.

(8) Busch-Petersen, J.; Corey, E. J. Tetrahedron Lett. 2000, 41, 2515.

(9) Feutrill, J. T.; Lilly, M. J.; Rizzacasa, M. A. Org. Lett. 2002, 4, 525. 